Article

\title{
Chemical Characterization and Antibacterial Activity of Essential Oil of Medicinal Plants from Eastern Serbia
}

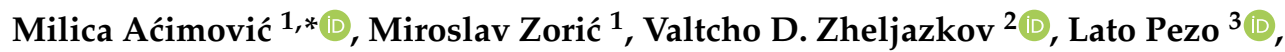 \\ Ivana Čabarkapa ${ }^{4}$, Jovana Stanković Jeremić ${ }^{5}$ and Mirjana Cvetković ${ }^{5}$ \\ 1 Institute of Field and Vegetable Crops Novi Sad, Maksima Gorkog 30, 21000 Novi Sad, Serbia; \\ miroslav.zoric@ifvens.ns.ac.rs \\ 2 College of Agricultural Sciences, Oregon State University, Corvallis, OR 97331, USA; \\ Valtcho.Jeliazkov@oregonstate.edu \\ 3 Institute of General and Physical Chemistry, University of Belgrade, Studentskitrg 12-16, \\ 11000 Belgrade, Serbia; latopezo@yahoo.co.uk \\ 4 Institute of Food Technology, University of Novi Sad, Bulevar Cara Lazara 1, 21000 Novi Sad, Serbia; \\ ivana.cabarkapa@fins.uns.ac.rs \\ 5 Institute of Chemistry, Technology and Metallurgy, University of Belgrade, Njegoševa 12, \\ 11000 Belgrade, Serbia; jovana_stankovic@hotmail.com (J.S.J.); miracvet13@gmail.com (M.C.) \\ * Correspondence: milica.acimovic@ifvens.ns.ac.rs
}

Received: 28 October 2020; Accepted: 18 November 2020; Published: 23 November 2020

\begin{abstract}
The objective of this study was to evaluate wild growing Satureja kitaibelii, Thymus serpyllum, Origanum vulgare, Achillea millefolium and Achillea clypeolata with respect to their essential oil (EO) content, composition and antimicrobial activity. The five species were collected at Mt. Rtanj and the village of Sesalac, Eastern Serbia. The main EO constituents of Lamiaceae plants were $p$-cymene $(24.4 \%)$, geraniol $(63.4 \%)$ and germacrene D $(21.5 \%)$ in Satureja kitaibelii, Thymus serpyllum and Origanum vulgare ssp. vulgare, respectively. A. millefolium $\mathrm{EO}$ had multiple constituents with major ones being camphor $(9.8 \%)$, caryophyllene oxide $(6.5 \%)$, terpinen-4-ol $(6.3 \%)$ and 1,8-cineole $(5.6 \%)$, while the main EO constituents of $A$. clypeolata were 1,8-cineole $(45.1 \%)$ and camphor $(18.2 \%)$. Antimicrobial testing of the EO showed that Staphylococcus aureus (Gram-positive) was more sensitive to all of the tested EOs than Escherichia coli (Gram-negative). S. kitaibelii EO showed the highest antimicrobial activity against both tested bacterial strains. This is the first study to characterize the EO composition and antimicrobial activity of these five medicinal species from Eastern Serbia in comparison with comprehensive literature data. The results can be utilized by the perfumery, cosmetics, food and pharmaceutical industries, but also for healing purposes in self-medication.
\end{abstract}

Keywords: Satureja kitaibelii; Thymus serpyllum; Origanum vulgare; Achillea millefolium; Achillea clypeolata

\section{Introduction}

The plants of the Lamiaceae and Asteraceae families are widely distributed medicinal plants throughout the world and have been used since ancient times for medicine and food. Historically, medicinal plants from these families have been used for flavoring, food preservation and medicinal purposes, due to their bioactive properties [1-3]. Today, pharmaceutical companies are interested in the wide range of beneficial properties of these plants in order to develop modern herbal remedies that would be used either as a replacement or supplement to conventional medicines and for prevention of illnesses [4-6]. In addition, medicinal plants are valuable raw material for perfumery and cosmetics, but also for healing purposes in self-medication [7-9], as well as in organic agriculture [10-12]. 
In Serbia, the Lamiaceae family includes the biggest number of medicinal plants, followed by Rosaceae and Asteraceae [13]. Satureja kitaibelii (Rtanj's tea), Thymus serpyllum (creeping thyme) and Origanum vulgare ssp. vulgare (common oregano) belong to the Lamiaceae family, while Achillea millefolium (yarrow) and A. clypeolata (moonshine yarrow) belong to the Asteraceae family. These plants are often utilized in Serbian traditional medicine as herbal teas for the treatment of urinary complaints and digestive disorders (diarrhea, abdominal cramping) [14-16]. These plants are also used externally for treating skin and mucous inflammation $[17,18]$.

Escherichia coli is the most dominant pathogen causing urinary tract infections [19-21] as well as foodborne illnesses [22-24], while Staphylococcus aureus is the most common cause for foodborne [25,26], skin and soft tissue infectious diseases [27-29]. However, over the past decade, the changing pattern of resistance in E. coli and S. aureus has emphasized the need for new antimicrobial agents [30-32]. Because of this, researchers are increasingly turning their attention towards traditional medicine. Therefore, there are a number of reports on some plant extracts and essential oils (EOs) with antimicrobial activity and as a source for antimicrobial agents against food spoilage and pathogens [33-35].

The aim of this study was to gather information on five medicinal plants from the Lamiaceae and Asteraceae family from Mt. Rtanj and the village of Sesalac in Eastern Serbia, to determine their EO yield and composition and compare the results with literature data on the same species. A second objective was to assess the antibacterial activity of these EOs against Staphylococcus aureus and Escherichia coli bacterial strains. Traditionally, the five plant species have been used by Serbian traditional medicine for treatment of various health conditions and diseases as outlined above.

\section{Results}

\subsection{Chemical Composition}

The most abundant compounds in S. kitaibelii EO were $p$-cymene (24.4\%), limonene (13.5\%) and linalool (8.3\%), and the most abundant ones in T. serpyllum EO were geraniol (63.4\%) and nerol (or cis-geraniol) (18.9\%), while in O. vulgare ssp. vulgare EO, the most abundant were germacrene D (21.5\%), 1,8-cineole (14.2\%), sabinene (14.0\%) and trans-caryophyllene (13.4\%). The most abundant constituents of $A$. millefolium EO were camphor (9.8\%), caryophyllene oxide (6.5\%), terpinen-4-ol $(6.3 \%)$ and 1,8 -cineole $(5.6 \%)$, while in the A. clypeolata EO, they were 1,8 -cineole $(45.1 \%)$ and camphor $(18.2 \%)$ (Table 1$)$.

Table 1. Chemical composition of investigated essential oils (EOs).

\begin{tabular}{ccccccccccc}
\hline & \multicolumn{2}{c}{ S. kitaibelii } & \multicolumn{2}{c}{ T. serpyllum } & $\begin{array}{c}\text { O. vulgare } \\
\text { ssp. vulgare }\end{array}$ & A. millefolium & \multicolumn{2}{c}{ A. clypeolata } \\
\hline Compound & RI & \% & RI & \% & RI & $\mathbf{\%}$ & RI & \% & RI & $\%$ \\
\hline Tricyclene & - & nd & - & nd & - & nd & - & nd & 927 & 0.1 \\
1-Octen-3-ol & - & nd & 981 & 0.2 & - & nd & - & nd & - & nd \\
$\alpha$-Thujene & 919 & 0.2 & - & nd & 915 & 0.1 & 928 & 0.2 & 929 & 0.1 \\
$\alpha$-Pinene & 927 & 2.5 & - & nd & 926 & 1.5 & 935 & 0.7 & 936 & 1.1 \\
Camphene & 945 & 1.1 & - & nd & 944 & 0.9 & 949 & 0.4 & 950 & 2.3 \\
Thuja-2,4(10)-diene & - & nd & - & nd & - & nd & - & nd & 955 & 0.1 \\
Sabinene & 966 & 0.2 & - & nd & 967 & 14.0 & 974 & 2.8 & 975 & 0.3 \\
$\beta$-Pinene & 971 & 0.5 & - & nd & 971 & 3.9 & 978 & 1.5 & 979 & 1.4 \\
Myrcene & 988 & 0.8 & - & nd & - & nd & - & nd & - & nd \\
dehydro-1,8-Cineole & - & nd & - & nd & - & nd & 991 & 0.2 & 993 & 0.1 \\
3-Octanol & 994 & 0.1 & - & nd & - & nd & - & nd & - & nd \\
$\alpha$-Phellandrene & 1005 & 0.2 & - & nd & - & nd & - & nd & - & nd \\
$\alpha$-Terpinene & 1015 & 0.9 & - & nd & - & nd & 1015 & 0.4 & 1016 & 0.6 \\
$p$-Cymene & 1022 & 24.4 & - & nd & - & nd & 1022 & 0.9 & 1022 & 3.1 \\
$\beta$-Phellandrene & - & nd & - & nd & 1026 & 1.6 & - & nd & - & nd \\
\hline
\end{tabular}


Table 1. Cont.

\begin{tabular}{|c|c|c|c|c|c|c|c|c|c|c|}
\hline \multirow[b]{2}{*}{ Compound } & \multicolumn{2}{|c|}{ S. kitaibelii } & \multicolumn{2}{|c|}{ T. serpyllum } & \multicolumn{2}{|c|}{$\begin{array}{l}\text { O. vulgare } \\
\text { ssp. vulgare }\end{array}$} & \multicolumn{2}{|c|}{ A. millefolium } & \multicolumn{2}{|c|}{ A. clypeolata } \\
\hline & RI & $\%$ & RI & $\%$ & RI & $\%$ & RI & $\%$ & RI & $\%$ \\
\hline Limonene & 1026 & 13.5 & - & nd & - & nd & 1027 & 0.1 & - & nd \\
\hline 1,8-Cineole & 1028 & 1.0 & - & nd & 1028 & 14.2 & 1028 & 5.6 & 1028 & 45.1 \\
\hline cis- $\beta$-Ocimene & 1032 & 2.3 & - & nd & 1033 & 6.8 & - & nd & - & nd \\
\hline trans- $\beta$-Ocimene & 1042 & 1.8 & - & nd & 1043 & 4.5 & - & nd & - & nd \\
\hline$\gamma$-Terpinene & 1052 & 3.3 & - & nd & 1052 & 0.4 & 1052 & 1.1 & 1053 & 1.2 \\
\hline cis-Sabinene hydrate & 1060 & 2.4 & - & nd & - & nd & 1060 & 0.4 & - & nd \\
\hline Terpinolene & 1081 & 0.4 & - & nd & - & nd & 1080 & 0.3 & - & nd \\
\hline$p$-Mentha-2,4(8)-diene & - & nd & - & nd & - & nd & - & nd & 1081 & 0.2 \\
\hline Linalool & 1092 & 8.3 & 1098 & 0.2 & - & nd & 1092 & 4.2 & 1092 & 0.4 \\
\hline n-Nonanal & - & nd & - & nd & - & nd & 1096 & 0.2 & - & nd \\
\hline cis-p-Menth-2-en-1-ol & 1112 & 0.3 & - & nd & - & nd & 1113 & 0.2 & 1114 & 0.2 \\
\hline Chrysanthenone & - & nd & - & nd & - & nd & 1116 & 0.2 & - & nd \\
\hline Nerol oxide & - & nd & 1155 & 0.1 & - & nd & - & nd & - & nd \\
\hline$\alpha$-Campholenal & 1118 & 0.1 & - & nd & - & nd & - & nd & 1119 & 0.2 \\
\hline trans-Pinocarveol & - & nd & - & nd & - & nd & 1132 & 0.1 & - & nd \\
\hline trans-p-Menth-2-en-1ol & - & nd & - & nd & - & nd & 1133 & 0.1 & - & nd \\
\hline Camphor & 1137 & 0.4 & - & nd & - & nd & 1138 & 9.8 & 1136 & 18.2 \\
\hline Pinocarvone & - & nd & - & nd & - & nd & 1155 & 0.7 & - & nd \\
\hline cis-Chrysanthenol & - & nd & - & nd & - & nd & - & nd & 1156 & 0.3 \\
\hline Borneol & 1160 & 4.9 & 1167 & 0.1 & 1160 & 1.2 & 1159 & 1.6 & 1159 & 2.7 \\
\hline$\delta$-Terpineol & - & nd & - & nd & - & nd & - & nd & 1161 & 0.6 \\
\hline cis-Pinocamphone & - & nd & - & nd & - & nd & 1167 & 0.1 & - & nd \\
\hline Menthol & 1167 & 0.1 & - & nd & - & nd & - & nd & - & nd \\
\hline Terpinen-4-ol & 1172 & 3.4 & - & nd & 1172 & 0.3 & 1173 & 6.3 & 1172 & 2.8 \\
\hline Thuj-3-en-10-al & - & nd & - & nd & - & nd & 1179 & 0.1 & - & nd \\
\hline p-Cymen-8-ol & 1181 & 0.2 & - & nd & - & nd & - & nd & - & nd \\
\hline $\begin{array}{l}\text { trans- } p \text {-Mentha- } \\
\text { 1(7),8-dien-2-ol }\end{array}$ & 1184 & 0.1 & - & nd & - & nd & - & nd & - & nd \\
\hline$\alpha$-Terpineol & 1187 & 0.4 & - & nd & 1187 & 0.7 & 1186 & 1.3 & 1186 & 2.4 \\
\hline Myrtenol & - & nd & - & nd & - & nd & 1189 & 0.2 & - & nd \\
\hline Myrtenal & - & nd & - & nd & - & nd & 1191 & 0.4 & 1192 & 0.3 \\
\hline cis-Dihydro carvone & 1193 & 0.7 & - & nd & - & nd & - & nd & - & nd \\
\hline trans-Dihydro carvone & 1201 & 1.8 & - & nd & - & nd & - & nd & - & nd \\
\hline trans-Carveol & 1216 & 0.3 & - & nd & - & nd & - & nd & - & nd \\
\hline Isobornylformate & 1225 & 0.1 & - & nd & - & nd & - & nd & - & nd \\
\hline cis-Carveol & - & nd & - & nd & - & nd & 1226 & 0.1 & - & nd \\
\hline $\begin{array}{l}\text { trans-Chrysanthenyl } \\
\text { acetate }\end{array}$ & - & nd & - & nd & - & nd & 1231 & 0.2 & - & nd \\
\hline Nerol & - & nd & 1233 & 18.9 & - & nd & - & nd & - & nd \\
\hline Cumin aldehyde & 1237 & 0.2 & - & nd & - & nd & 1235 & 0.1 & - & nd \\
\hline Thymol, methyl ether & - & nd & 1239 & 0.2 & - & nd & - & nd & - & nd \\
\hline Carvacrol, methyl ether & 1241 & 0.7 & - & nd & - & nd & - & nd & - & nd \\
\hline Neral & - & nd & 1245 & 0.7 & - & nd & - & nd & - & nd \\
\hline $\begin{array}{l}\text { cis-Chrysanthenyl } \\
\text { acetate }\end{array}$ & - & nd & - & nd & - & nd & 1257 & 0.4 & - & nd \\
\hline Geraniol & - & nd & 1258 & 63.4 & - & nd & - & nd & - & nd \\
\hline Geranial & - & nd & 1275 & 1.2 & - & nd & - & nd & - & nd \\
\hline Bornyl acetate & - & nd & - & nd & 1286 & 0.1 & 1282 & 0.4 & - & nd \\
\hline Thymol & 1291 & 0.2 & 1298 & 0.1 & - & nd & 1288 & 0.6 & 1290 & 0.9 \\
\hline Carvacrol & 1299 & 2.3 & - & nd & - & nd & 1298 & 1.1 & 1300 & 0.5 \\
\hline$p$-Mentha-1,4,-dien-7-ol & - & nd & - & nd & - & nd & 1325 & 0.2 & - & nd \\
\hline Methyl geranate & - & nd & 1328 & 0.2 & - & nd & - & nd & - & nd \\
\hline trans-Carvyl acetate & - & nd & - & nd & - & nd & 1334 & 0.1 & - & nd \\
\hline Eugenol & - & nd & - & nd & - & nd & 1354 & 0.1 & - & nd \\
\hline cis-Carvyl acetate & - & nd & - & nd & - & nd & 1359 & 0.1 & - & nd \\
\hline
\end{tabular}


Table 1. Cont.

\begin{tabular}{|c|c|c|c|c|c|c|c|c|c|c|}
\hline \multirow[b]{2}{*}{ Compound } & \multicolumn{2}{|c|}{ S. kitaibelii } & \multicolumn{2}{|c|}{ T. serpyllum } & \multicolumn{2}{|c|}{$\begin{array}{l}\text { O. vulgare } \\
\text { ssp. vulgare }\end{array}$} & \multicolumn{2}{|c|}{ A. millefolium } & \multicolumn{2}{|c|}{ A. clypeolata } \\
\hline & RI & $\%$ & RI & $\%$ & RI & $\%$ & RI & $\%$ & RI & $\%$ \\
\hline Neryl acetate & - & nd & 1368 & 0.3 & - & nd & - & nd & - & nd \\
\hline$\alpha$-Copaene & 1371 & 0.3 & - & nd & - & nd & 1373 & 0.1 & - & nd \\
\hline$\beta$-Bourbonene & 1383 & 0.9 & - & nd & 1384 & 1.9 & 1387 & 0.1 & - & nd \\
\hline Geranyl acetate & - & nd & 1388 & 4.7 & - & nd & - & nd & - & nd \\
\hline$\beta$-Elemene & 1390 & 0.2 & - & nd & - & nd & - & nd & - & nd \\
\hline cis-Jasmone & - & nd & - & nd & - & nd & 1395 & 0.1 & - & nd \\
\hline Methyl eugenol & - & nd & - & nd & - & nd & 1401 & 0.1 & - & nd \\
\hline trans-Caryophyllene & 1418 & 2.8 & 1425 & 4.6 & 1420 & 13.4 & 1418 & 4.7 & 1417 & 0.5 \\
\hline$\beta$-Copaene & 1428 & 0.2 & - & nd & - & nd & - & nd & - & nd \\
\hline cis- $\beta$-Farnesene & - & nd & - & nd & - & nd & 1441 & 0.1 & - & nd \\
\hline$\alpha$-Humulene & 1453 & 0.1 & 1459 & 0.2 & 1454 & 2.1 & 1441 & 0.1 & - & nd \\
\hline trans- $\beta$-Farnesene & - & nd & - & nd & - & nd & 1455 & 0.1 & - & nd \\
\hline allo-Aromadendrene & - & nd & - & nd & - & nd & - & nd & 1458 & 1.3 \\
\hline 9-epi-trans-Caryophyllene & - & nd & - & nd & 1461 & 0.5 & 1459 & 0.4 & - & nd \\
\hline$\gamma$-Muurolene & 1476 & 0.1 & - & nd & - & nd & 1473 & 0.3 & - & nd \\
\hline Germacrene D & 1481 & 3.6 & 1487 & 0.3 & 1486 & 21.5 & 1480 & 2.9 & 1484 & 1.2 \\
\hline$\beta$-Selinene & - & nd & - & nd & - & nd & 1484 & 0.2 & - & nd \\
\hline $\begin{array}{l}\text { trans-Muurola- } \\
4(14), 5 \text {-diene }\end{array}$ & - & nd & - & nd & - & nd & 1490 & 0.1 & - & nd \\
\hline epi-Cubebol & - & nd & - & nd & - & nd & 1493 & 0.5 & - & nd \\
\hline Bicyclogermacrene & 1497 & 1.4 & - & nd & 1497 & 1.7 & - & nd & - & nd \\
\hline$\beta$-Bisabolene & 1508 & 1.0 & 1515 & 2.0 & - & nd & - & nd & - & nd \\
\hline $\begin{array}{c}\text { (trans, } \\
\text { trans)- } \alpha \text {-Farnesene }\end{array}$ & - & nd & - & nd & 1509 & 0.7 & - & nd & - & nd \\
\hline$\gamma$-Cadinene & - & nd & - & nd & - & nd & 1512 & 1.1 & 1512 & 1.2 \\
\hline$\delta$-Cadinene & 1523 & 0.2 & - & nd & 1524 & 1.1 & - & nd & 1522 & 0.2 \\
\hline$\alpha$-Calacorene & - & nd & - & nd & - & nd & 1540 & 0.3 & - & nd \\
\hline Elemol & - & nd & - & nd & - & nd & 1546 & 0.7 & - & nd \\
\hline trans-Nerolidol & - & nd & - & nd & - & nd & 1560 & 0.1 & - & nd \\
\hline Geranyl butanoate & - & nd & 1565 & 0.2 & - & nd & - & nd & - & nd \\
\hline Germacrene D-4-ol & - & nd & - & nd & 1575 & 0.8 & - & nd & - & nd \\
\hline ar-Tumerol & - & nd & - & nd & - & nd & 1575 & 0.6 & - & nd \\
\hline Spathulenol & 1575 & 2.0 & - & nd & 1577 & 0.6 & - & nd & 1573 & 0.4 \\
\hline Caryophyllene oxide & 1581 & 2.9 & 1587 & 0.9 & 1583 & 3.9 & 1580 & 6.5 & 1582 & 3.2 \\
\hline Viridiflorol & - & nd & - & nd & - & nd & 1587 & 0.5 & - & nd \\
\hline Salvial-4(14)-en-1-one & 1591 & 0.1 & - & nd & - & nd & - & nd & - & nd \\
\hline Ledol & - & nd & - & nd & - & nd & 1596 & 0.1 & - & nd \\
\hline Geranyl isovalerate & - & nd & 1606 & 0.2 & - & nd & - & nd & - & nd \\
\hline $\begin{array}{c}\text { Humulene epoxide II + } \\
\beta \text {-Oplopenone }\end{array}$ & - & nd & - & nd & 1606 & 0.3 & 1603 & 0.6 & 1603 & 0.2 \\
\hline $\begin{array}{l}\text { Muurola-4,10(14)- } \\
\text { dien-1- } \beta \text {-ol }\end{array}$ & - & nd & - & nd & - & nd & - & nd & 1622 & 0.3 \\
\hline$\gamma$-Eudesmol & - & nd & - & nd & - & nd & 1626 & 1.5 & - & nd \\
\hline $\begin{array}{l}\text { Caryophylla-4(12),8(13)- } \\
\text { dien-5- } \alpha \text {-ol }\end{array}$ & - & nd & 1640 & 0.4 & - & nd & 1631 & 1.8 & 1630 & 0.7 \\
\hline epi- $\alpha$-Cadinol & - & nd & - & nd & - & nd & - & nd & 1635 & 2.4 \\
\hline $\begin{array}{l}\text { epi- } \alpha \text {-Murrolol } \\
\text { (=tau-muurolol) }\end{array}$ & - & nd & - & nd & 1640 & 0.4 & 1636 & 3.3 & - & nd \\
\hline$\beta$-Eudesmol & - & nd & - & nd & - & nd & 1646 & 2.4 & - & nd \\
\hline$\alpha$-Cadinol & - & nd & - & nd & 1654 & 0.9 & 1648 & 1.2 & 1648 & 1.5 \\
\hline $\begin{array}{l}\text { 14-hydroxy-9-epi- } \\
\text { trans-Caryophyllene }\end{array}$ & 1669 & 0.3 & - & nd & - & nd & - & nd & - & nd \\
\hline$\alpha$-Bisabolol & - & nd & - & nd & - & nd & 1678 & 0.3 & - & nd \\
\hline
\end{tabular}


Table 1. Cont.

\begin{tabular}{|c|c|c|c|c|c|c|c|c|c|c|}
\hline \multirow[b]{2}{*}{ Compound } & \multicolumn{2}{|c|}{ S. kitaibelii } & \multicolumn{2}{|c|}{ T. serpyllum } & \multicolumn{2}{|c|}{$\begin{array}{l}\text { O. vulgare } \\
\text { ssp. vulgare }\end{array}$} & \multicolumn{2}{|c|}{ A. millefolium } & \multicolumn{2}{|c|}{ A. clypeolata } \\
\hline & RI & $\%$ & RI & $\%$ & RI & $\%$ & RI & $\%$ & RI & $\%$ \\
\hline $\begin{array}{c}\text { Germacra- } 4(15), 5,10(14) \\
\text {-trien-1- } \alpha \text {-ol }\end{array}$ & - & nd & - & nd & - & nd & 1681 & 1.7 & - & nd \\
\hline $\begin{array}{l}\text { Eudesma-4(15),7- } \\
\text { dien-1- } \beta \text {-ol }\end{array}$ & 1684 & 0.4 & - & nd & - & nd & - & nd & - & nd \\
\hline Curcuphenol & - & nd & - & nd & - & nd & 1711 & 0.2 & - & nd \\
\hline 2-cis,6-trans Farnesol & - & nd & - & nd & - & nd & 1715 & 0.2 & - & nd \\
\hline Chamazulene & - & nd & - & nd & - & nd & 1724 & 0.1 & - & nd \\
\hline 6R,7R-Bisabolone & - & nd & - & nd & - & nd & 1739 & 1.2 & - & nd \\
\hline$\beta$-Costol & - & nd & - & nd & - & nd & 1761 & 0.3 & - & nd \\
\hline $\begin{array}{l}\text { 6,10,14-trimethyl- } \\
\text { 2-Pentadecanone }\end{array}$ & - & nd & - & nd & - & nd & 1840 & 0.2 & - & nd \\
\hline Heptadecanal & - & nd & - & nd & - & nd & 1913 & 0.1 & - & nd \\
\hline Heneicosane & - & nd & - & nd & - & nd & 2100 & 0.2 & - & nd \\
\hline Phytol & - & nd & - & nd & - & nd & 2123 & 0.1 & - & nd \\
\hline $\begin{array}{l}(\text { cis, cis })-9,12- \\
\text { Octadecadienoic acid }\end{array}$ & - & nd & - & nd & - & nd & 2148 & 0.1 & - & nd \\
\hline trans-Geranylgeraniol & - & nd & - & nd & - & nd & 2172 & 0.3 & - & nd \\
\hline Tricosane & - & nd & - & nd & - & nd & 2301 & 0.6 & - & nd \\
\hline Pentacosane & - & nd & - & nd & - & nd & 2497 & 0.4 & - & nd \\
\hline Heptacosane & 2701 & 0.1 & - & nd & - & nd & 2701 & 0.2 & - & nd \\
\hline Nonacosane & 2896 & 0.1 & 2895 & 0.1 & - & nd & 2909 & 0.3 & - & nd \\
\hline Total & & 96.8 & & 99.2 & & 100.0 & & 80.5 & & 98.3 \\
\hline
\end{tabular}

RI-retention index on a HP-5MS column; nd-not detected.

\subsection{Antibacterial Activity}

According to the assay, S. aureus was more sensitive to the tested EOs than E. coli. However, S. kitaibelii expressed the most potent activity against both G-positive and G-negative bacteria, followed by T. serpyllum and A. clypeolata, while O. vulgare ssp. vulgare showed the weakest antibacterial activity. A. millefolium showed weak activity against $E$. coli but was very potent against $S$. aureus (Table 2).

Table 2. Antimicrobial activity of tested EO against Escherichia coli and Staphylococcus aureus ( $\mu \mathrm{L} / \mathrm{mL})$.

\begin{tabular}{ccccc}
\hline & \multicolumn{2}{c}{$\begin{array}{c}\text { Escherichia coli (ATCC8739) } \\
\text { G-Negative }\end{array}$} & \multicolumn{2}{c}{$\begin{array}{c}\text { Staphylococcus aureus (ATCC25923) } \\
\text { G-Positive }\end{array}$} \\
\hline & MIC & MBC & MIC & MBC \\
\hline S. kitaibelii & $0.156 \pm 0.013^{\mathrm{a}}$ & $0.312 \pm 0.023^{\mathrm{a}}$ & $0.078 \pm 0.002^{\mathrm{a}}$ & $0.156 \pm 0.005^{\mathrm{a}}$ \\
T. serpyllum & $20.00 \pm 0.52^{\mathrm{c}}$ & $10.00 \pm 0.05^{\mathrm{b}}$ & $2.50 \pm 0.19^{\mathrm{c}}$ & $5.00 \pm 0.21^{\mathrm{c}}$ \\
O. vulgare ssp. & $20.00 \pm 0.43^{\mathrm{c}}$ & $>20.00^{\mathrm{d}}$ & $10.00 \pm 0.03^{\mathrm{d}}$ & $20.00 \pm 0.43^{\mathrm{d}}$ \\
vulgare & & $>20.00^{\mathrm{d}}$ & $1.25 \pm 0.10^{\mathrm{b}}$ & $2.50 \pm 0.17^{\mathrm{b}}$ \\
A. millefolium & $20.00 \pm 0.23^{\mathrm{c}}$ & $20.00 \pm 0.41^{\mathrm{c}}$ & $2.50 \pm 0.09^{\mathrm{c}}$ & $5.00 \pm 0.04^{\mathrm{c}}$ \\
A. clypeolata & $10.00 \pm 0.24^{\mathrm{b}}$ & ${ }^{\mathrm{c}}$ &
\end{tabular}

Means in the same column with different superscript are statistically different, according to Tukey's HSD test $(p \leq 0.05) ; n=10$ repetitions; MIC-minimal inhibitory concentration; MBC-minimal bactericidal concentration.

Further investigations will be focused on studying major constituents of S. kitaibelii, T. serpyllum, O. vulgare ssp. vulgare, A. millefolium and A. clypeolata EOs, such as geraniol, 1,8-cineole, $p$-cymene, germacrene $\mathrm{D}$, nerol, camphor, sabinene, limonene, trans-caryophyllene, etc., to evaluate concentrations of the components that could be responsible for the antibacterial effect. Additional tests with a larger number of bacteria regarding the synergic potential of EOs will be implemented in our future investigations. 


\section{Discussion}

\subsection{Satureja kitaibelii}

S. kitaibelii EO constituents from this study and from literature reports [18,36-40] are shown in Table 3. The classification between them according to the content of chemical compounds was performed using HCA analysis (Figure 1a). This analysis showed that differences in EO composition could be separated into several potential clusters (chemotypes): geraniol, $p$-cymene and limonene, with high abundance of linalool and borneol, which is in accordance with a previous study [18].

Table 3. The essential oil constituents of $S$. kitaibelii from this study and from literature reports.

\begin{tabular}{|c|c|c|c|c|c|c|c|c|c|c|c|c|c|c|}
\hline Locality & Ref. & 岕 & 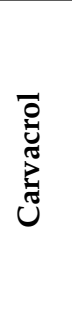 & 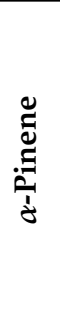 & 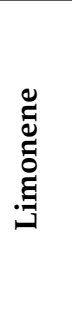 & 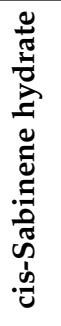 & 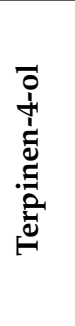 & 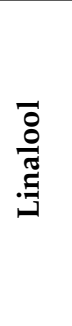 & $\begin{array}{l}\overline{0} \\
\text { छٌ } \\
0\end{array}$ & 串 & 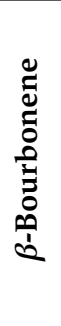 & 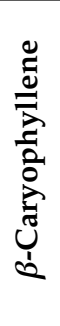 & 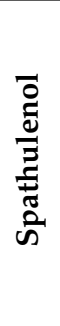 & 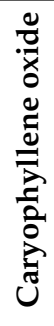 \\
\hline Rtanj & TS & 24.4 & 2.3 & 2.5 & 13.5 & 2.4 & 3.4 & 8.3 & 4.9 & 0.0 & 0.9 & 0.0 & 2.0 & 2.9 \\
\hline Rtanj & [36] & 33.6 & 14.1 & 1.1 & 8.5 & 5.5 & 1.2 & 3.0 & 3.6 & 0.0 & 0.0 & 0.0 & 2.1 & 2.3 \\
\hline Rtanj & [37] & 22.3 & 2.9 & 4.3 & 11.4 & 1.1 & 2.3 & 5.9 & 7.6 & 0.0 & 0.0 & 1.5 & 2.4 & 1.7 \\
\hline Suva planina (JK) & [38] & 20.9 & 0.0 & 6.0 & 16.0 & 8.2 & 3.8 & 1.6 & 9.8 & 0.0 & 0.0 & 0.0 & 0.0 & 0.0 \\
\hline Staraplanina $(\mathrm{V})$ & [39] & 34.2 & 0.0 & 0.0 & 10.5 & 0.0 & 0.0 & 5.0 & 10.4 & 0.0 & 0.0 & 0.0 & 0.0 & 3.0 \\
\hline Đerdap & [18] & 21.9 & 0.0 & 2.4 & 8.0 & 9.0 & 3.8 & 0.0 & 7.7 & 0.0 & 1.5 & 2.2 & 2.9 & 5.9 \\
\hline Vlaškaplanina (P) & [18] & 16.9 & 0.0 & 3.2 & 7.4 & 3.2 & 2.5 & 22.2 & 7.6 & 0.0 & 1.9 & 1.2 & 1.2 & 1.6 \\
\hline Suva planina (DG) & [40] & 4.3 & 0.0 & 0.6 & 7.9 & 5.1 & 0.0 & 5.8 & 3.7 & 28.1 & 0.0 & 0.0 & 2.1 & 5.0 \\
\hline Suva planina (DG) & [39] & 7.7 & 0.0 & 0.0 & 6.1 & 0.0 & 0.0 & 12.0 & 0.0 & 23.2 & 0.0 & 0.0 & 0.0 & 4.3 \\
\hline Suva planina (SK) & [40] & 1.4 & 0.0 & 1.1 & 4.3 & 3.8 & 0.0 & 14.8 & 1.8 & 30.3 & 0.3 & 0.0 & 2.8 & 5.2 \\
\hline Šljivovički vis & [39] & 4.4 & 0.0 & 0.0 & 5.7 & 0.0 & 0.0 & 9.5 & 5.4 & 23.2 & 0.0 & 0.0 & 0.0 & 0.0 \\
\hline Svrljiškeplanine & [39] & 9.3 & 0.0 & 0.0 & 7.9 & 0.0 & 10.8 & 10.7 & 0.0 & 13.4 & 0.0 & 0.0 & 0.0 & 0.0 \\
\hline Staraplanina (VR) & {$[40]$} & 1.4 & 0.0 & 0.8 & 5.9 & 0.7 & 0.0 & 5.0 & 2.4 & 24.0 & 0.0 & 0.0 & 1.5 & 4.4 \\
\hline Staraplanina (GS) & [39] & 7.5 & 0.0 & 0.0 & 0.0 & 0.0 & 4.1 & 6.2 & 0.0 & 29.7 & 0.0 & 4.5 & 0.0 & 0.0 \\
\hline Staraplanina $(\mathrm{T})$ & [18] & 5.5 & 0.0 & 2.0 & 5.2 & 2.0 & 3.2 & 0.3 & 7.7 & 12.0 & 5.9 & 4.2 & 5.8 & 3.8 \\
\hline Staraplanina (GK) & [39] & 6.6 & 0.0 & 0.0 & 0.0 & 0.0 & 10.3 & 6.5 & 9.4 & 13.0 & 0.0 & 0.0 & 0.0 & 0.0 \\
\hline
\end{tabular}

Ref.-reference; TS-this study; JK-Jelasnicka Klisura; V-Vetren; P_Poganovo; DG-Devojacki Grob; SK—Sicevacka Klisura; VR_Visoka Rzana; GS—Gornja Sokolovica; T—Temsica; GK-Golemei Kamen.

Taking into account the chemical content range, the correlation network based on components from S. kitaibelii EO is shown in Figure 1b. There were strong positive correlations between $p$-cymene and limonene $(\mathrm{r}=0.75 ; p<0.01)$, as well as between $\alpha$-pinene and sabinene hydrate $(\mathrm{r}=0.76 ; p<0.01)$. However, the strongest negative correlation was between geraniol and compounds such as $p$-cymene $(\mathrm{r}=-0.86 p<0.01)$, limonene $(\mathrm{r}=-0.74 ; p<0.01)$ and borneol $(\mathrm{r}=-0.66 ; p<0.01)$.

The major constituent of extracts and EO used in traditional medicines as an antimicrobial agent is $p$-cymene [41]. However, in this study, $p$-cymene exhibited the weakest antibacterial activity against $S$. aureus and E. coli [42]. Contrarily, limonene exhibited moderate antibacterial activity against these bacteria [43], while linalool exhibited strong antimicrobial activity [44]. It is well known that many plants exert their beneficial effects through the additive or synergistic action of several chemical compounds acting at single or multiple target sites [45]. The results from this study suggest that the combination effects of these compounds ( $p$-cymene, limonene and linalool) in S. kitaibelii EO had 
antibacterial enhancement (synergistic or additive effects) against tested bacteria (S. aureus and E. coli). Further investigations into this topic need to be conducted.

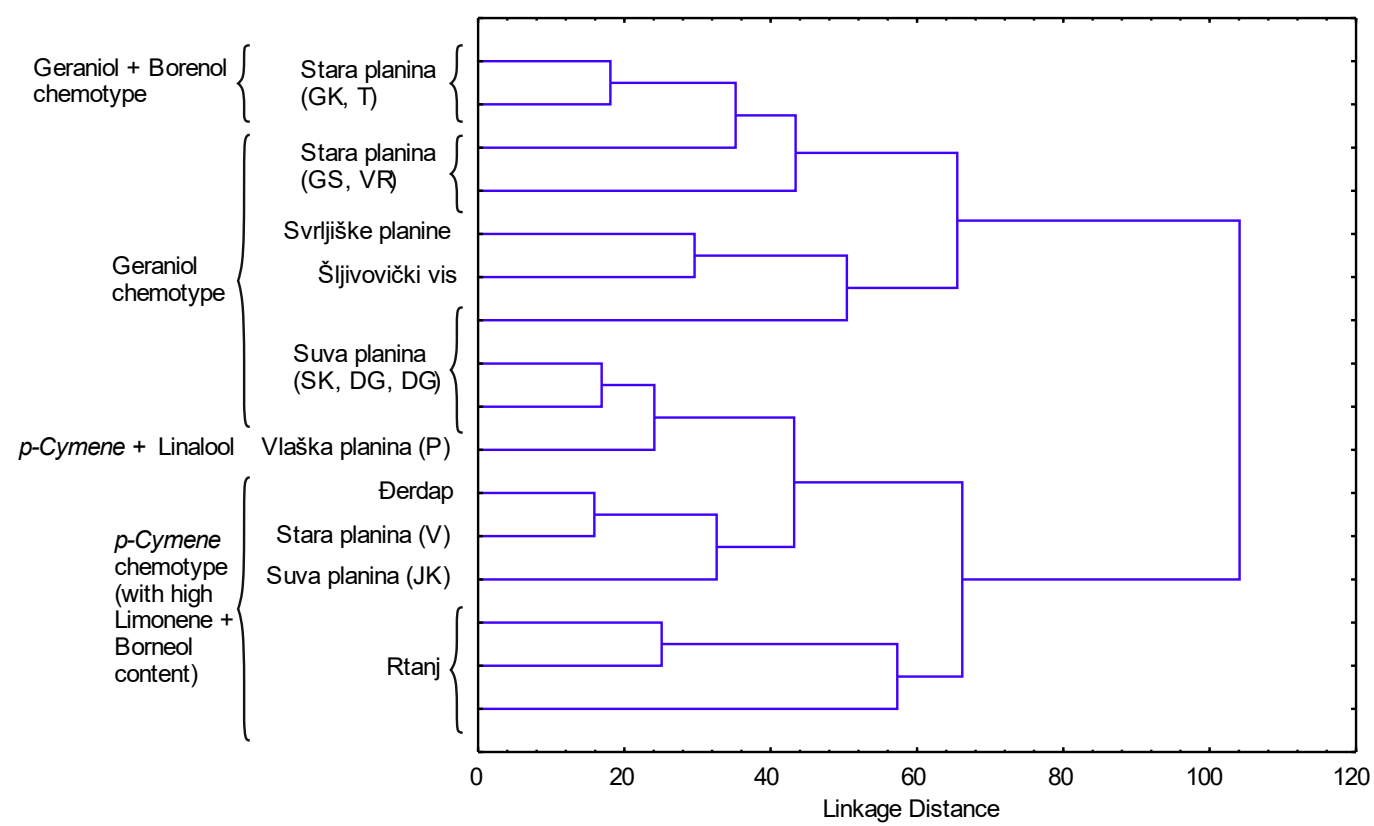

(a)

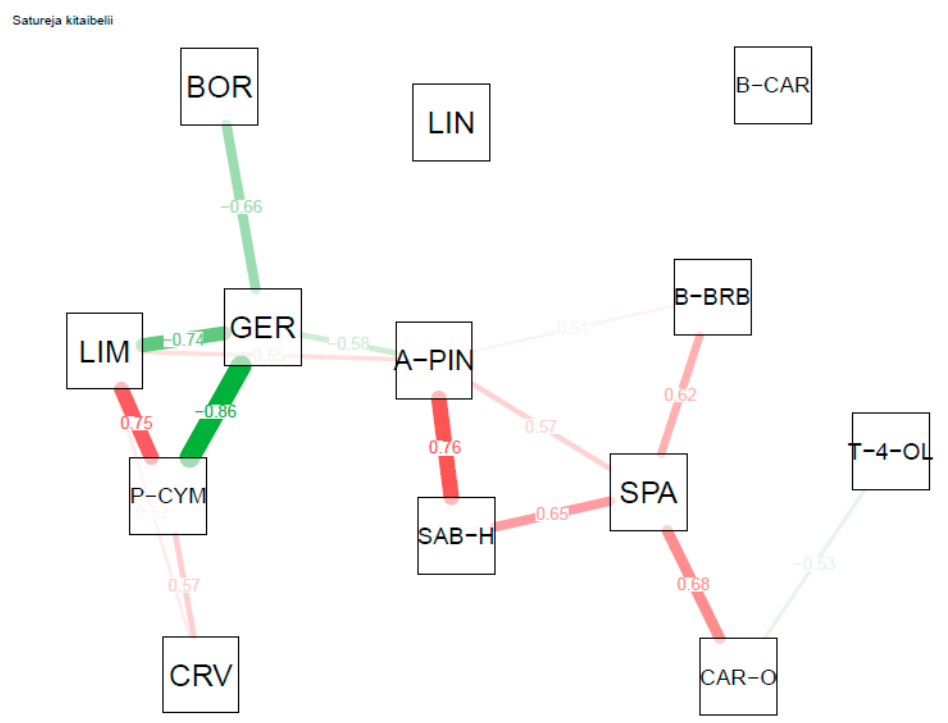

(b)

Figure 1. (a) Dendogram of the EO constituents of S. kitaibelii from this study and from literature reports (the samples are marked according to Table 3); (b) correlation network based on S. kitaibelii EO constituents (P-CYM-p-cymene; CRV-Carvacrol; A-PIN— $\alpha$-pinene; LIM-limonene; SAB-H—cis-sabinene hydrate; T-4-OL—terpinen-4-ol; LIN-linalool; BOR-borneol; GER—geraniol; B-BRB- $\beta$-bourbonene; B-CAR— $\beta$-caryophyllene; SPA—spathulenol; CAR-O—caryophyllene oxide).

\subsection{Thymus serpyllum}

Differentiation between T. serpyllum EO samples from this study and from literature reports [46-57] (Table 4) was performed using HCA analysis (Figure 2a). Several clusters (potential chemotypes) were established, including thymol, carvacrol, linalool (linalool and linalyl acetate), geraniol (geraniol and geranyl acetate) and terpinene (terpinene and terpinene acetate), as well as a number of multiple-component chemotypes [46,58]. Research conducted on 20 accessions of T. serpyllum in 
Estonia showed the presence of three different chemotypes: trans-nerolidol + caryophyllene oxide, trans-nerolidol + myrcene and myrcene chemotypes [52]. Furthermore, variability between 16 populations from Poland showed three chemotypes: geranyl acetate $+\beta$-terpineol $+\beta$-myrcene, geranyl acetate $+\beta$-terpineol + borneol and pure linalool chemotype [47].

Table 4. The essential oil constituents of other T. serpyllum reported in the literature.

\begin{tabular}{|c|c|c|c|c|c|c|c|c|c|c|c|c|c|c|}
\hline Locality & Ref. & 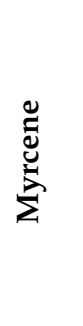 & 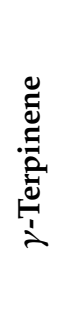 & $\begin{array}{l}\overline{0} \\
\text { Z } \\
\text { Z }\end{array}$ & 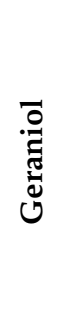 & $\underset{\mathrm{z}}{\overrightarrow{0}}$ & J & 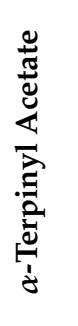 & 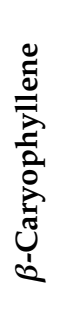 & 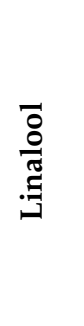 & 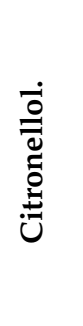 & 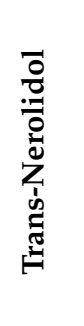 & 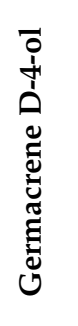 & 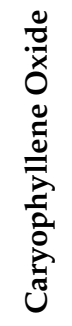 \\
\hline Serbia, Rtanj & TS & 0.0 & 0.0 & 18.9 & 63.4 & 0.1 & 0.0 & 0.0 & 4.6 & 0.2 & 0.0 & 0.0 & 0.3 & 0.9 \\
\hline Kazakhstan 4 & [46] & 0.0 & 1.4 & 2.8 & 55.9 & 5.1 & 1.2 & 0.0 & 0.0 & 0.0 & 0.0 & 0.0 & 0.0 & 0.0 \\
\hline Poland 13 & [47] & 1.0 & 0.6 & 3.9 & 0.0 & 0.2 & 1.0 & 0.0 & 0.0 & 0.7 & 23.9 & 0.3 & 0.0 & 1.0 \\
\hline Poland 8 & [47] & 0.6 & 0.5 & 0.0 & 1.0 & 0.1 & 0.2 & 0.0 & 0.0 & 56.6 & 0.7 & 0.3 & 0.0 & 0.2 \\
\hline Serbia, Zlatar (G) & [48] & 8.2 & 0.2 & 0.0 & 0.0 & 0.2 & 0.0 & 66.2 & 2.7 & 0.2 & 0.0 & 0.0 & 0.5 & 0.6 \\
\hline Serbia, Kopaonik (B) & [49] & 3.8 & 1.8 & 0.0 & 0.0 & 0.1 & 0.0 & 0.0 & 33.3 & 0.0 & 0.0 & 0.1 & 0.1 & 1.7 \\
\hline Serbia, Kopaonik (BB) & {$[50]$} & 0.0 & 1.4 & 0.0 & 0.0 & 5.6 & 0.0 & 0.0 & 27.7 & 1.2 & 0.0 & 2.4 & 1.1 & 1.3 \\
\hline Serbia, Pasjača (Ž) & [51] & 1.6 & 1.5 & 0.0 & 1.4 & 7.3 & 0.6 & 1.0 & 2.8 & 0.7 & 0.0 & 24.2 & 16.0 & 1.1 \\
\hline Estonia 3 & {$[52]$} & 0.8 & 0.0 & 0.0 & 0.0 & 0.5 & 0.3 & 0.0 & 3.8 & 0.0 & 0.0 & 52.0 & 3.3 & 4.2 \\
\hline Estonia 6 & {$[52]$} & 0.2 & 0.0 & 0.0 & 0.0 & 2.9 & 1.7 & 0.0 & 3.1 & 0.0 & 0.0 & 49.5 & 3.8 & 11.2 \\
\hline Estonia 8 & [52] & 0.0 & 0.0 & 0.0 & 0.0 & 1.0 & 0.3 & 0.0 & 1.8 & 0.0 & 0.0 & 70.1 & 2.8 & 4.5 \\
\hline Estonia 9 & [52] & 0.3 & 0.0 & 0.0 & 0.0 & 0.5 & 0.3 & 0.0 & 5.7 & 0.0 & 0.0 & 20.5 & 5.3 & 6.4 \\
\hline Estonia 13 & [52] & 8.6 & 0.0 & 0.0 & 0.0 & 1.4 & 2.0 & 0.0 & 4.6 & 0.0 & 0.0 & 24.3 & 7.9 & 2.5 \\
\hline Estonia 15 & [52] & 6.4 & 0.0 & 0.0 & 0.0 & 1.0 & 0.8 & 0.0 & 5.4 & 0.0 & 0.0 & 34.5 & 7.2 & 5.7 \\
\hline Estonia 17 & [52] & 15.3 & 0.0 & 0.0 & 0.0 & 1.0 & 1.1 & 0.0 & 5.4 & 0.0 & 0.0 & 33.1 & 6.2 & 1.5 \\
\hline Estonia 18 & [52] & 10.7 & 0.0 & 0.0 & 0.0 & 0.5 & 0.5 & 0.0 & 2.2 & 0.0 & 0.0 & 30.0 & 3.1 & 1.4 \\
\hline Estonia 19 & [52] & 6.9 & 0.0 & 0.0 & 0.0 & 0.8 & 0.8 & 0.0 & 11.7 & 0.0 & 0.0 & 27.6 & 10.5 & 7.4 \\
\hline Estonia 2 & [52] & 0.1 & 0.0 & 0.0 & 0.0 & 0.2 & 0.1 & 0.0 & 7.0 & 0.0 & 0.0 & 30.1 & 6.6 & 24.0 \\
\hline Estonia 5 & [52] & 0.0 & 0.0 & 0.0 & 0.0 & 0.8 & 0.5 & 0.0 & 3.4 & 0.0 & 0.0 & 32.9 & 4.3 & 25.0 \\
\hline Estonia 7 & [52] & 0.6 & 0.0 & 0.0 & 0.0 & 1.0 & 0.5 & 0.0 & 3.5 & 0.0 & 0.0 & 27.6 & 1.7 & 20.8 \\
\hline Estonia 10 & [52] & 0.0 & 0.0 & 0.0 & 0.0 & 0.9 & 0.3 & 0.0 & 13.3 & 0.0 & 0.0 & 28.4 & 6.8 & 16.4 \\
\hline Estonia 4 & [52] & 0.0 & 0.0 & 0.0 & 0.0 & 1.9 & 0.9 & 0.0 & 4.6 & 0.0 & 0.0 & 4.8 & 5.9 & 45.0 \\
\hline Poland 1 & [53] & 1.1 & 9.0 & 0.0 & 0.0 & 0.1 & 43.0 & 0.0 & 6.9 & 0.5 & 0.2 & 0.0 & 2.1 & 0.8 \\
\hline Poland 2 & {$[54]$} & 1.8 & 10.8 & 0.0 & 0.0 & 0.2 & 44.9 & 0.0 & 0.0 & 0.9 & 0.1 & 0.0 & 1.3 & 0.8 \\
\hline Kazakhstan 1 & [46] & 0.0 & 13.5 & 0.0 & 0.0 & 13.2 & 55.2 & 0.0 & 1.4 & 0.0 & 0.0 & 0.0 & 0.0 & 0.0 \\
\hline Kazakhstan 3 & [46] & 0.0 & 15.7 & 0.0 & 0.0 & 11.3 & 55.9 & 0.0 & 0.0 & 0.0 & 0.0 & 0.0 & 0.0 & 0.0 \\
\hline India, Kumaon & [55] & 0.5 & 3.4 & 0.0 & 0.0 & 58.8 & 1.0 & 0.0 & 1.2 & 0.2 & 0.0 & 0.0 & 0.0 & 0.4 \\
\hline Serbia, Čačak & [56] & 0.0 & 0.0 & 0.0 & 1.2 & 37.4 & 0.4 & 0.0 & 0.0 & 0.0 & 0.0 & 0.0 & 6.7 & 0.0 \\
\hline Kazakhstan 6 & [46] & 0.0 & 14.6 & 0.0 & 0.0 & 58.3 & 6.2 & 0.0 & 0.0 & 0.0 & 0.0 & 0.0 & 0.0 & 0.0 \\
\hline
\end{tabular}


Table 4. Cont.

\begin{tabular}{|c|c|c|c|c|c|c|c|c|c|c|c|c|c|c|}
\hline Locality & Ref. & 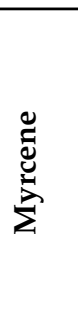 & 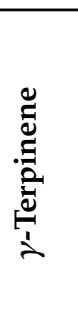 & $\begin{array}{l}\overline{0} \\
\text { Z } \\
\text { Z }\end{array}$ & ত゚ & $\underset{\mathrm{E}}{\stackrel{0}{\mathrm{E}}}$ & 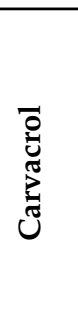 & 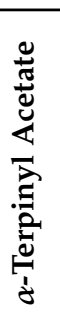 & 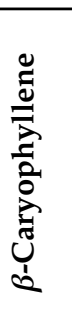 & 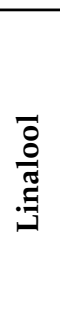 & 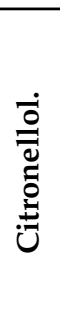 & 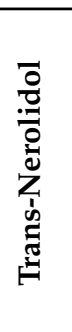 & 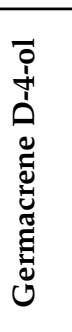 & 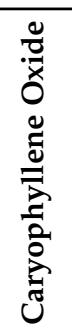 \\
\hline Kazakhstan 7 & [46] & 0.0 & 15.1 & 0.0 & 0.0 & 44.3 & 6.1 & 0.0 & 0.0 & 0.0 & 0.0 & 0.0 & 0.0 & 0.0 \\
\hline India & [57] & 0.1 & 1.9 & 0.5 & 0.0 & 17.7 & 11.8 & 0.0 & 0.0 & 0.0 & 0.0 & 0.0 & 0.0 & 0.0 \\
\hline Kazakhstan 2 & [46] & 0.9 & 13.9 & 0.0 & 0.0 & 28.6 & 38.4 & 0.0 & 0.8 & 0.0 & 0.0 & 0.0 & 0.0 & 0.0 \\
\hline Kazakhstan 5 & {$[46]$} & 0.0 & 13.5 & 0.0 & 0.0 & 31.7 & 25.7 & 0.0 & 1.3 & 0.0 & 0.0 & 0.0 & 0.0 & 0.0 \\
\hline Kazakhstan 8 & [46] & 1.0 & 16.5 & 0.0 & 0.0 & 24.2 & 35.3 & 0.0 & 0.7 & 0.0 & 0.0 & 0.0 & 0.0 & 0.0 \\
\hline Estonia 1 & [52] & 1.4 & 0.0 & 0.0 & 0.0 & 0.0 & 0.1 & 0.0 & 8.6 & 0.0 & 0.0 & 1.7 & 11.2 & 1.6 \\
\hline Estonia 11 & [52] & 11.2 & 0.0 & 0.0 & 0.0 & 0.9 & 0.6 & 0.0 & 11.0 & 0.0 & 0.0 & 4.2 & 3.3 & 10.8 \\
\hline Estonia 12 & [52] & 0.4 & 0.0 & 0.0 & 0.0 & 2.9 & 3.5 & 0.0 & 13.2 & 0.0 & 0.0 & 2.7 & 12.4 & 17.7 \\
\hline Estonia 14 & [52] & 20.2 & 0.0 & 0.0 & 0.0 & 0.8 & 0.7 & 0.0 & 9.4 & 0.0 & 0.0 & 15.2 & 10.2 & 2.2 \\
\hline Estonia 16 & [52] & 18.6 & 0.0 & 0.0 & 0.0 & 1.3 & 1.3 & 0.0 & 13.0 & 0.0 & 0.0 & 2.2 & 11.4 & 8.2 \\
\hline Estonia 20 & [52] & 10.5 & 0.0 & 0.0 & 0.0 & 1.1 & 1.5 & 0.0 & 10.5 & 0.0 & 0.0 & 6.0 & 11.2 & 9.2 \\
\hline Poland 10 & [47] & 10.5 & 0.8 & 0.1 & 0.4 & 8.4 & 1.6 & 0.0 & 0.0 & 1.8 & 0.1 & 1.3 & 0.0 & 2.0 \\
\hline Poland 11 & [47] & 8.3 & 1.0 & 0.0 & 0.0 & 0.0 & 0.2 & 0.0 & 0.0 & 0.9 & 0.1 & 0.1 & 0.0 & 0.4 \\
\hline Poland 12 & [47] & 5.2 & 0.8 & 0.1 & 0.0 & 0.3 & 0.6 & 0.0 & 0.0 & 3.2 & 3.1 & 0.1 & 0.0 & 0.2 \\
\hline Poland 14 & [47] & 9.4 & 0.0 & 0.0 & 0.1 & 1.0 & 2.7 & 0.0 & 0.0 & 3 & 0.0 & 0.4 & 0.0 & 10.7 \\
\hline Poland 15 & [47] & 15.7 & 3.7 & 0.0 & 0.3 & 7.7 & 1.2 & 0.0 & 0.0 & 1.7 & 0.1 & 1.2 & 0.0 & 2.1 \\
\hline Poland 16 & [47] & 2.9 & 0.2 & 0.1 & 0.1 & 0.9 & 2.7 & 0.0 & 0.0 & 1.6 & 0.1 & 3.0 & 0.0 & 1.8 \\
\hline
\end{tabular}

Ref.-reference; TS—this study; G-Gradina; B-Brzeće; bb—Belo brdo; Ž—Žitorađa.

Data in Figure 2a suggests the presence of 11 potential chemotypes in T. serpyllum: geraniol, citronellol, linalool, $\alpha$-terpinyl acetate, trans-nerolidol, trans-nerolidol + caryophyllene oxide, caryophyllene oxide, carvacrol, thymol, thymol + carvacrol and multiple-component chemotypes. Previous research on T. vulgaris described genetically distinct chemotypes that can be distinguished on the basis of the dominant monoterpene produced in the glandular trichomes. It was established that the monoterpene variations in T. vulgaris plants may represent an adaptive strategy in relation to the environmental variations, as the different chemotypes showed different geographic and locality distribution [59].

Taking into account the chemical content range, the correlation network based on components from T. serpyllum $\mathrm{EO}$ is shown in Figure $2 \mathrm{~b}$. There were strong negative multiple correlations between sabinene and $\beta$-caryophyllene, caryophyllene oxide and germacrene $\mathrm{D}(p<0.01)$, but all these three compounds are in positive correlations $(p<0.01)$.

Previous research reported that geraniol exhibited good antimicrobial activity and, in combination with antibiotics, would have substantial therapeutic potential against S. aureus and E. coli infections [60]. Although geraniol and nerol are geometric isomers, they demonstrated equal activities [61]. Taking into account the moderate antibacterial activity against S. aureus and E. coli, EOs of T. vulgaris (with geraniol and nerol) could be used as herbal supplements to conventional therapy. 


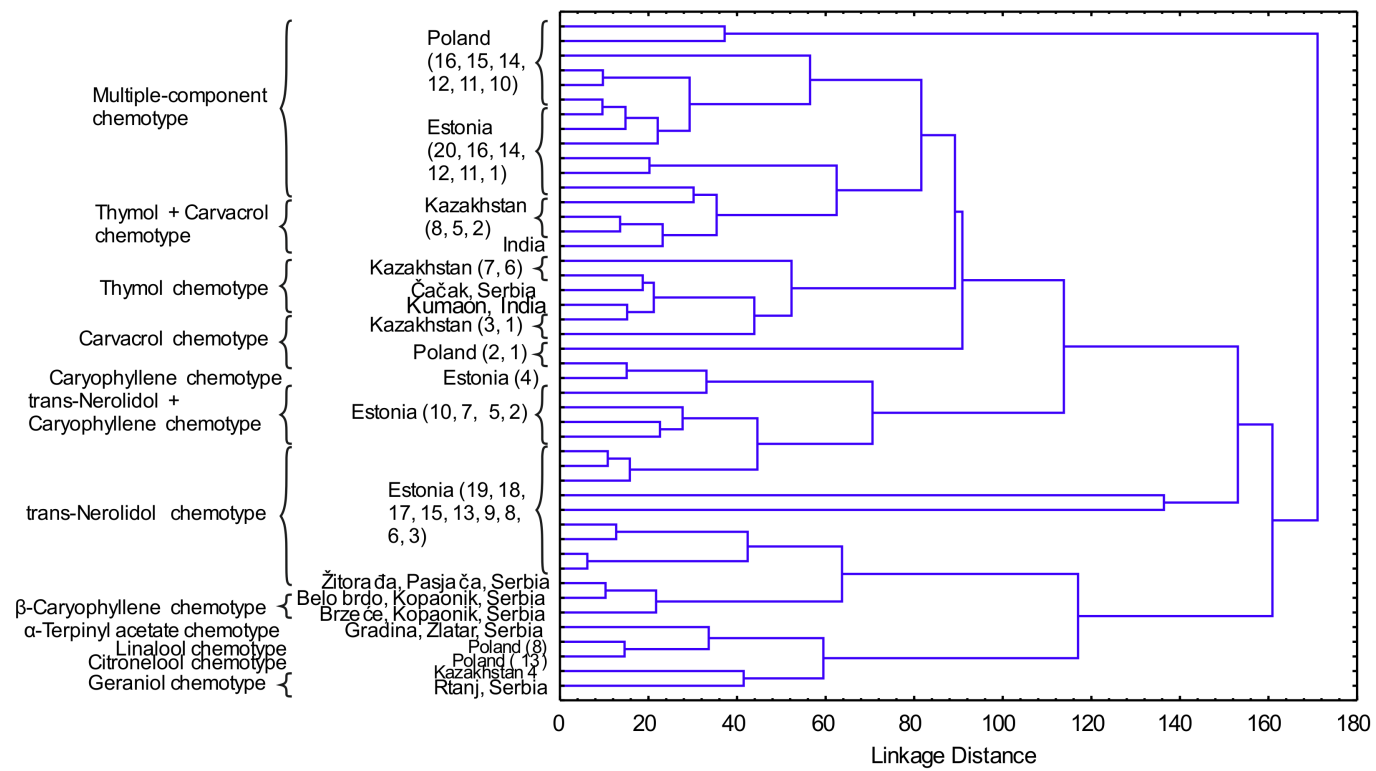

(a)

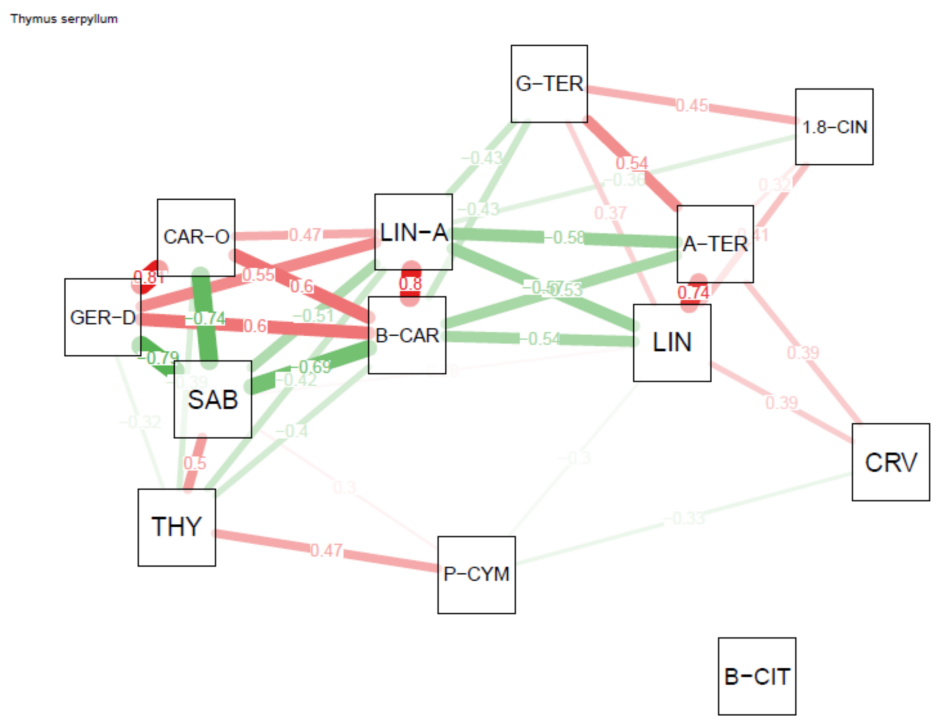

(b)

Figure 2. (a) Dendogram of the EO constituents of T. serpyllum from this study and from literature reports (the samples are marked according to Table 4); (b) correlation network based on T. serpyllum EO constituents (THY — thymol; CRV—carvacrol; MYR—-myrcene; G-TER— $\gamma$-terpinene; NER—nerol; GER—geraniol; LIN-linalool; CIT—citronellol; A-TRP— $\alpha$-terpinyl acetate; B-CAR— $\beta$-caryophyllene; GRM-germacrene; CAR-O—caryophyllene oxide; T-NER—trans-nerolidol).

\subsection{Origanum vulgare}

The classification between O. vulgare ssp. vulgare EO samples from this study and from literature reports [62-73] according to the content of chemical compounds (Table 5) was performed using HCA analysis (Figure 3a), which showed several clusters. O. vulgare ssp. vulgare collected at Mt. Rtanj can be classified as germacrene D chemotype [65]. The latter authors mentioned three more chemotypes: sabinene, $\beta$-ocimene and $\beta$-caryophyllene. The results showed differences among the oregano accessions with respect to morphological traits and chemical constituents of EOs, indicating the existence of infraspecific variations and chemical polymorphism [74]. Chemical composition of aerial parts of O. vulgare collected during the flowering season (August 2011), on Mt. Mokra Gora, Southwestern 
Serbia, showed the presence of sabinene (10.2\%), terpinen-4-ol (9.3\%), 1,8 cineole (5.8\%), $\gamma$-terpinene $(5.6 \%)$ and caryophyllene oxide (5.4\%) as main compounds [75]. However, another study on O. vulgare in Serbia showed the presence of thymol (45\%) and carvacrol (37.4\%) as the major EO constituents [76].

Table 5. O. vulgare ssp. vulgare EO constituents from this study and others reported previously in the literature.

\begin{tabular}{|c|c|c|c|c|c|c|c|c|c|c|c|c|c|c|}
\hline Locality & Ref. & $\begin{array}{l}\stackrel{\Xi}{\Xi} \\
\stackrel{\Xi}{\tilde{N}}\end{array}$ & 导 & 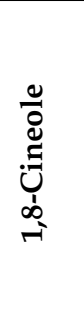 & 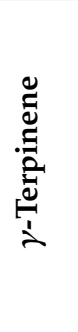 & 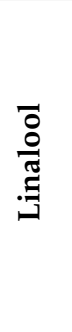 & $\frac{\bar{d}}{\stackrel{d}{2}}$ & 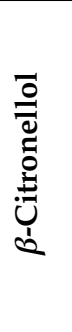 & 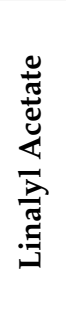 & 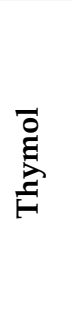 & 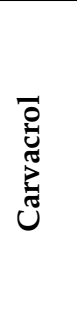 & 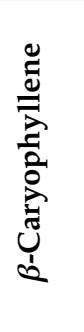 & 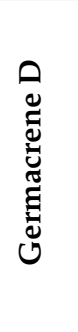 & 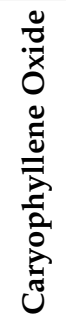 \\
\hline Serbia, Rtanj & TS & 14.0 & 0.0 & 14.2 & 0.4 & 0.0 & 0.7 & 0.0 & 0.0 & 0.0 & 0.0 & 13.4 & 21.5 & 3.9 \\
\hline Montenegro, Radovče & [62] & 3.6 & 1.2 & 3.2 & 0.6 & 4.9 & 5.4 & 0.0 & 0.6 & 1.2 & 0.2 & 14.4 & 27.9 & 1.8 \\
\hline Montenegro, Lipovo & [62] & 5.9 & 4.6 & 2.9 & 1.4 & 6.6 & 4.8 & 0.0 & 0.0 & 0.2 & 1.0 & 12.7 & 15.4 & 2.2 \\
\hline Montenegro, Grahovo & {$[62]$} & 4.6 & 3.7 & 3.3 & 2.8 & 3.1 & 5.7 & 0.0 & 0.5 & 0.0 & 0.0 & 14.6 & 16.8 & 2.7 \\
\hline Moldova & {$[63]$} & 9.8 & 0.7 & 0.3 & 2.1 & 0.8 & 0.6 & 0.0 & 0.0 & 0.3 & 11.7 & 13.1 & 17.0 & 1.6 \\
\hline Lithuania & {$[64]$} & 7.4 & 0.2 & 8.5 & 0.1 & 11.3 & 2.7 & 0.0 & 0.0 & 0.2 & 0.1 & 10.0 & 13.2 & 3.4 \\
\hline Albania, n3 & {$[64]$} & 7.3 & 0.6 & 3.4 & 1.8 & 0.3 & 2.3 & 0.0 & 0.0 & 2.6 & 0.2 & 11.1 & 18.6 & 1.2 \\
\hline Lithuania, Silenai & [65] & 8.7 & 0.2 & 6.1 & 1.9 & 1.4 & 1.5 & 0.0 & 0.0 & 0.0 & 0.0 & 12.7 & 14.1 & 2.1 \\
\hline Lithuania, Rastinenai & [65] & 10.1 & 0.8 & 6.4 & 1.6 & 1.8 & 1.5 & 0.0 & 0.0 & 0.0 & 0.0 & 12.6 & 14.2 & 4.4 \\
\hline China, Kunlun Mt & [66] & 0.0 & 0.0 & 0.0 & 0.0 & 0.4 & 0.0 & 85.3 & 0.0 & 0.0 & 0.0 & 0.4 & 0.0 & 0.0 \\
\hline Pakistan & [66] & 0.0 & 0.3 & 0.0 & 0.0 & 0.4 & 0.0 & 72.7 & 0.0 & 7.2 & 0.0 & 1.0 & 0.0 & 0.4 \\
\hline China, Hetian & [66] & 0.0 & 0.0 & 0.0 & 0.0 & 0.5 & 0.0 & 75.0 & 0.0 & 0.0 & 0.0 & 0.5 & 0.2 & 0.5 \\
\hline China, Shangqiu & [66] & 0.0 & 7.4 & 0.0 & 1.9 & 0.4 & 0.0 & 0.0 & 0.0 & 42.9 & 0.0 & 7.8 & 0.3 & 2.2 \\
\hline Iran, Noshahr & {$[67]$} & 0.8 & 3.6 & 3.8 & 9.7 & 0.3 & 1.2 & 0.0 & 0.0 & 37.1 & 9.6 & 1.9 & 1.1 & 0.7 \\
\hline China, Anhui & {$[66]$} & 0.5 & 0.9 & 20.8 & 0.4 & 5.5 & 0.0 & 0.0 & 0.0 & 1.5 & 0.0 & 10.2 & 0.5 & 2.5 \\
\hline Czech & [64] & 6.3 & 1.1 & 17.4 & 0.0 & 2.0 & 1.7 & 0.0 & 0.3 & 0.0 & 0.0 & 6.0 & 6.1 & 13.5 \\
\hline China, Yili & {$[66]$} & 0.0 & 0.0 & 0.0 & 0.0 & 3.2 & 3.9 & 0.0 & 0.0 & 0.0 & 0.0 & 17.7 & 9.8 & 32.9 \\
\hline Iran, MeshkinSahahr & [68] & 2.2 & 0.0 & 1.4 & 12.3 & 0.0 & 0.0 & 0.0 & 0.0 & 0.0 & 8.0 & 1.2 & 0.0 & 21.0 \\
\hline Finland & {$[64]$} & 2.3 & 7.2 & 3.3 & 3.7 & 0.0 & 0.3 & 0.0 & 0.0 & 0.0 & 4.6 & 7.9 & 5.5 & 11.4 \\
\hline Montenegro, Boljevići & [62] & 0.9 & 2.0 & 0.8 & 3.1 & 8.8 & 17.8 & 0.0 & 9.7 & 8.3 & 1.1 & 7.7 & 16.0 & 0.5 \\
\hline Spain & {$[64]$} & 0.4 & 0.0 & 0.0 & 0.0 & 3.2 & 52.8 & 0.0 & 0.0 & 0.0 & 0.0 & 6.4 & 11.3 & 1.3 \\
\hline Montenegro, Kameno & [62] & 0.0 & 7.8 & 0.0 & 6.5 & 0.0 & 0.2 & 0.0 & 0.0 & 0.0 & 74.3 & 1.3 & 0.2 & 0.0 \\
\hline Iran, Kaleybar & {$[68]$} & 0.9 & 0.0 & 0.0 & 17.5 & 0.0 & 0.0 & 0.0 & 0.0 & 0.0 & 21.3 & 11.3 & 1.5 & 2.7 \\
\hline Croatia & {$[64]$} & 0.0 & 2.6 & 0.0 & 1.6 & 0.0 & 0.1 & 0.0 & 0.0 & 0.3 & 86.0 & 0.4 & 0.0 & 0.2 \\
\hline Portugal, n7 & {$[64]$} & 0.4 & 5.1 & 0.0 & 9.8 & 0.6 & 0.1 & 0.0 & 0.4 & 0.3 & 26.5 & 9.3 & 4.6 & 2.9 \\
\hline Iran, Gardrahmat & [69] & 0.0 & 0.0 & 0.0 & 20.5 & 0.0 & 0.0 & 0.0 & 0.0 & 15.4 & 23.5 & 5.1 & 0.0 & 2.1 \\
\hline Iran, Khezr Abad & [69] & 0.0 & 0.0 & 0.0 & 18.4 & 0.0 & 0.0 & 0.0 & 0.0 & 0.0 & 59.4 & 0.0 & 0.0 & 0.0 \\
\hline Iran, Perdanan & [69] & 0.0 & 0.0 & 0.0 & 9.6 & 0.0 & 0.0 & 0.0 & 0.0 & 0.0 & 58.5 & 3.7 & 0.0 & 0.3 \\
\hline Iran, Garderan & [69] & 0.0 & 0.0 & 0.0 & 7.7 & 0.0 & 0.0 & 0.0 & 0.0 & 0.0 & 67.1 & 0.0 & 0.0 & 0.4 \\
\hline
\end{tabular}


Table 5. Cont.

\begin{tabular}{|c|c|c|c|c|c|c|c|c|c|c|c|c|c|c|}
\hline Locality & Ref. & & 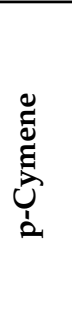 & 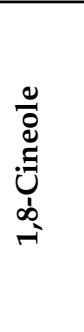 & 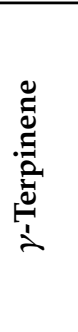 & 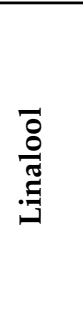 & 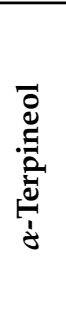 & 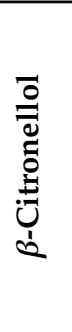 & 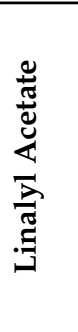 & 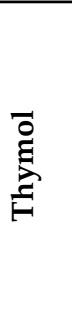 & 总 & 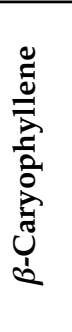 & 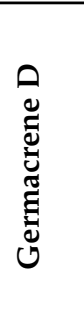 & 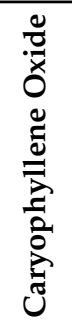 \\
\hline Iran, Sabalan & [68] & 20.8 & 0.0 & 0.0 & 7.1 & 0.0 & 0.0 & 0.0 & 0.0 & 0.0 & 17.8 & 6.1 & 0.0 & 3.1 \\
\hline Poland, Drohiczyn 1 & [70] & 25.4 & 1.7 & 0.9 & 3.3 & 2.7 & 0.8 & 0.0 & 0.0 & 2.1 & 0.5 & 11.5 & 14.2 & 3.0 \\
\hline Poland, Radecznica & [70] & 25.9 & 2.1 & 2.2 & 2.5 & 3.4 & 1.7 & 0.0 & 0.0 & 1.1 & 1.8 & 14.8 & 11.2 & 2.4 \\
\hline Poland, Lupkow & [70] & 14.5 & 7.4 & 1.5 & 6.1 & 3.3 & 1.3 & 0.0 & 0.0 & 1.7 & 1.7 & 10.8 & 11.8 & 1.3 \\
\hline Poland, Javornik & {$[70]$} & 19.9 & 2.3 & 11.9 & 3.7 & 6.0 & 3.5 & 0.0 & 0.0 & 1.1 & 1.9 & 9.7 & 8.4 & 1.0 \\
\hline Poland, Zwierzyniec & {$[70]$} & 17.1 & 4.4 & 10.0 & 5.6 & 4.0 & 1.7 & 0.0 & 0.0 & 0.5 & 0.1 & 9.8 & 2.9 & 10.5 \\
\hline Poland, Babice & {$[70]$} & 15.4 & 5.1 & 8.3 & 1.5 & 14.1 & 3.2 & 0.0 & 0.0 & 2.0 & 2.2 & 10.1 & 0.6 & 3.4 \\
\hline Poland, Czarnystok & [70] & 21.6 & 11.0 & 4.5 & 6.5 & 4.9 & 0.7 & 0.0 & 0.0 & 0.6 & 0.6 & 8.3 & 7.2 & 3.7 \\
\hline Poland, Monasterzec & [70] & 15.0 & 2.9 & 2.3 & 8.3 & 10.4 & 1.7 & 0.0 & 0.0 & 2.0 & 1.3 & 11.8 & 5.1 & 2.7 \\
\hline Iran, Chalus & [68] & 3.2 & 0.0 & 2.8 & 16.5 & 0.0 & 0.0 & 0.0 & 0.0 & 0.0 & 6.4 & 1.4 & 0.0 & 0.0 \\
\hline Poland, Lukowe & {$[70]$} & 12.1 & 1.5 & 14.7 & 1.8 & 1.1 & 4.6 & 0.0 & 0.0 & 0.9 & 2.0 & 18.2 & 9.6 & 2.1 \\
\hline Poland, Drohiczyn 2 & [70] & 6.2 & 12.7 & 1.6 & 1.6 & 4.1 & 1.9 & 0.0 & 0.0 & 1.0 & 1.9 & 21.3 & 1.9 & 9.0 \\
\hline Latvia & [64] & 0.0 & 0.0 & 0.0 & 0.0 & 0.0 & 0.0 & 0.0 & 0.0 & 0.0 & 0.0 & 25.1 & 19.4 & 13.1 \\
\hline Turkey & [71] & 1.6 & 0.1 & 2.1 & 0.6 & 1.9 & 2.2 & 0.0 & 1.9 & 0.0 & 2.3 & 20.9 & 17.8 & 0.0 \\
\hline Poland, Lipowiec & [70] & 6.5 & 8.6 & 8.9 & 0.2 & 32.1 & 3.3 & 0.0 & 0.0 & 1.4 & 0.7 & 3.5 & 0.2 & 10.0 \\
\hline Poland, Karlikow & [70] & 8.1 & 8.5 & 7.9 & 1.4 & 15.0 & 3.8 & 0.0 & 0.0 & 0.8 & 1.5 & 17.8 & 1.5 & 3.9 \\
\hline Poland, Bukowsko & [70] & 1.9 & 5.9 & 0.2 & 5.7 & 24.2 & 1.1 & 0.0 & 0.0 & 1.2 & 1.6 & 19.6 & 5.9 & 2.4 \\
\hline Poland, Gruszka & [70] & 7.4 & 4.5 & 0.4 & 6.2 & 16.0 & 1.2 & 0.0 & 0.0 & 1.5 & 0.8 & 10.1 & 4.7 & 2.5 \\
\hline Portugal, n8 & {$[64]$} & 0.1 & 0.2 & 0.0 & 0.6 & 84.7 & 0.3 & 0.0 & 0.0 & 0.0 & 4.7 & 1.5 & 2.4 & 0.1 \\
\hline Albania, n7 & {$[64]$} & 2.3 & 0.4 & 1.5 & 1.0 & 1.3 & 0.8 & 0.0 & 33.0 & 2.8 & 0.2 & 9.0 & 20.6 & 0.3 \\
\hline Iran, Ilam & {$[72]$} & 1.5 & 0.0 & 5.4 & 0.9 & 1.4 & 0.0 & 0.0 & 0.0 & 25.3 & 12.3 & 0.0 & 0.1 & 0.0 \\
\hline India, Srinagar & [73] & 15.3 & 23.9 & 0.0 & 38.4 & 0.0 & 0.0 & 0.0 & 0.0 & 0.0 & 0.9 & 1.2 & 1.2 & 0.0 \\
\hline India, Pulwama & [73] & 18.1 & 21.1 & 0.0 & 25.7 & 0.0 & 0.0 & 0.0 & 0.0 & 0.1 & 14.8 & 3.0 & 1.7 & 0.0 \\
\hline India, Tangmarg & [73] & 6.5 & 33.3 & 0.0 & 8.4 & 0.0 & 0.0 & 0.0 & 0.0 & 0.8 & 27.2 & 0.9 & 0.6 & 0.0 \\
\hline
\end{tabular}

Correlation network based on O. vulgare ssp. vulgare EO constituents is shown in Figure 3b. There were two groups of correlations between O. vulgare ssp. vulgare EO constituents. Compounds in positive correlations were predominantly germacrene $\mathrm{D}, \beta$-caryophyllene, caryophyllene oxide, $\alpha$-terpinene, 1,8-cineole, linalool, sabinene, $p$-cymene, thymol and linalyl acetate. On the other hand, compounds with predominantly negative correlations were $\beta$-citronellol, carvacrol and $\gamma$-terpinene $(p<0.01)$, with the last two having a strong positive correlation $(\mathrm{r}=0.66, p<0.01)$.

An antimicrobial effect of synthetic antibiotic in combination with germacrene D showing growth inhibition on E. coli and S. aureus [77] is noted. Conversely, 1,8-cineole derivatives displayed significant antibacterial activity [78], while trans-caryophyllene displayed moderate antibacterial activity [79]. Sabinene exhibited prominent antibiofilm properties against E. coli and S. aureus providing a novel and effective alternative/complementary approach to counteract chronic infections and the transmission of 
diseases in clinical settings [80]. The weak antibacterial activity of $O$. vulgare ssp. vulgare EO could be attributed to low concentrations of bioactive compounds and their inability to exhibit activity.

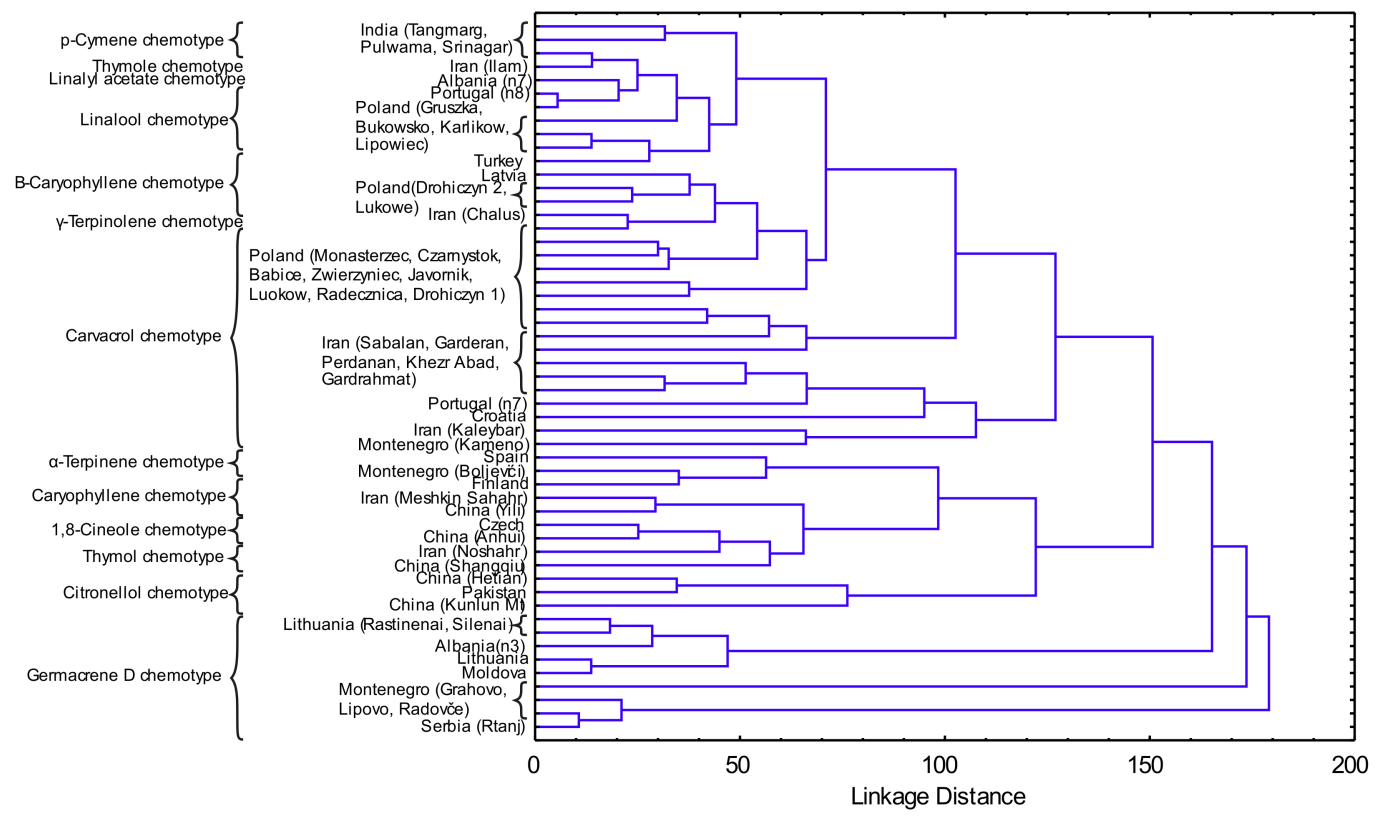

(a)

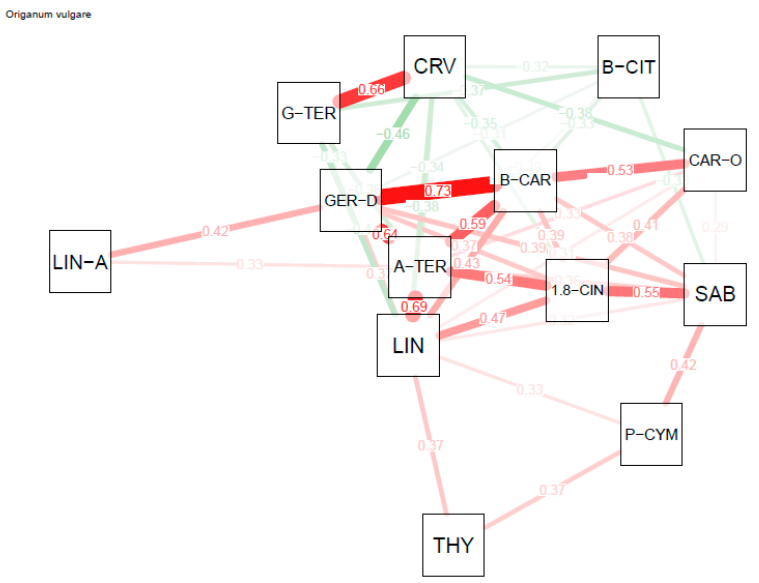

(b)

Figure 3. (a) Dendogram of the EO constituents of $O$. vulgarefrom this study and from literature reports (the samples are marked according to Table 5); (b) correlation network based on O. vulgare EO constituents (P-CYM- $p$-cymene; THY—thymol; CRV—carvacrol; SAB—sabinene; G-TER- $\gamma$-terpinene; 1,8-CIN-1,8-cineole; LIN-linalool; A-TER— $\alpha$-terpineol; B-CIT- $\beta$-citronellol; LIN-A—linalyl acetate; $\mathrm{B}-\mathrm{CAR}$ - $\beta$-caryophyllene; GER-D—germacrene D; CAR-O—caryophyllene oxide).

\subsection{Achillea millefolium}

A. millefolium represents a polyploidic complex of hardly distinguishable species, subspecies, forms and hybrids [81]. However, morphological, chemical and molecular traits as well as PCA analysis showed that the terpenoid variation can be used to explore biogenetic pathways [82]. The differentiation between A. millefolium EO from this study and from literature reports [83-92] (Table 6) was performed using HCA analysis (Figure 4a). Several clusters (potential chemotypes) were established, including camphor, lavandulyl acetate, sabinene, 1,8-cineole, chrysanthenyl acetate, $\beta$-pinene and chamazulene chemotypes. 
Table 6. A. millefolium EO constituents from this study and others reported previously in the literature.

\begin{tabular}{|c|c|c|c|c|c|c|c|c|c|c|c|c|c|c|}
\hline Locality & Ref. & 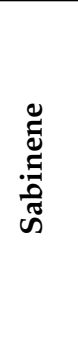 & 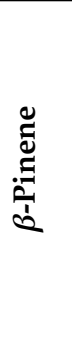 & $\underset{0}{\stackrel{0}{0}}$ & $\frac{\tilde{a}}{\tilde{z}}$ & $\begin{array}{l}\overline{0} \\
\stackrel{d}{0} \\
0 \\
0\end{array}$ & 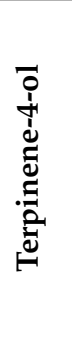 & 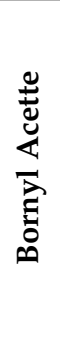 & 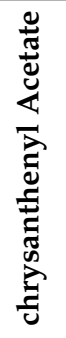 & 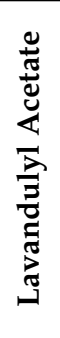 & 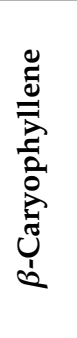 & 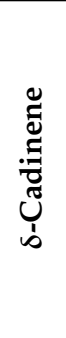 & 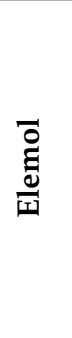 & 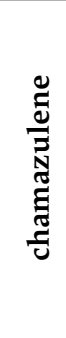 \\
\hline $\begin{array}{l}\text { India, Himachal } \\
\text { Pradesh }\end{array}$ & [83] & 17.6 & 6.3 & 13.0 & 0.0 & 12.4 & 6.2 & 8.0 & & 0.0 & 2.3 & & 0.0 & 5.3 \\
\hline Serbia, Busilovac & [84] & 16.9 & 5.1 & 10.1 & 7.1 & 1.6 & 12.9 & 0.0 & 3.0 & 3.4 & 3.3 & 0.2 & 0.0 & 0.4 \\
\hline Serbia, Sokobanja & [84] & 1.1 & 21.3 & 7.3 & 3.7 & 19.5 & 1.8 & 0.1 & 0.0 & 0.0 & 2.8 & 0.7 & 0.6 & 0.0 \\
\hline Serbia, Sokobanja2 & [84] & 0.9 & 13.2 & 11.1 & 0.5 & 6.7 & 1.1 & 0.0 & 0.0 & 0.2 & 2.5 & 0.7 & 9.9 & 0.0 \\
\hline Serbia, Ražanj & {$[84]$} & 2.0 & 16.3 & 7.8 & 1.8 & 0.6 & 0.5 & 0.0 & 0.0 & 0.3 & 3.4 & 1.6 & 14.7 & 0.0 \\
\hline Serbia, Ravna Gora & {$[84]$} & 4.6 & 23.6 & 16.9 & 0.4 & 0.0 & 0.6 & 0.0 & 0.0 & 0.0 & 8.3 & 0.2 & 0.0 & 10.5 \\
\hline Serbia, Topli do & [84] & 4.5 & 26.7 & 17.1 & 1.0 & 2.8 & 0.5 & 0.0 & 0.0 & 0.0 & 9.4 & 0.3 & 0.0 & 7.4 \\
\hline Serbia, Dobro polje & {$[84]$} & 3.4 & 36.3 & 18.4 & 1.1 & 0.0 & 0.9 & 0.0 & 0.0 & 0.0 & 5.9 & 0.2 & 0.0 & 1.7 \\
\hline Serbia, Rajac1 & [84] & 5.7 & 18.7 & 10.8 & 1.3 & 0.0 & 0.8 & 0.0 & 0.0 & 0.0 & 16.0 & 0.0 & 0.0 & 1.3 \\
\hline Serbia, Medvednik & [84] & 4.9 & 24.8 & 10.5 & 3.9 & 1.7 & 0.6 & 0.0 & 0.0 & 0.0 & 5.7 & 0.5 & 1.1 & 10.1 \\
\hline Serbia, Rajac2 & {$[84]$} & 3.0 & 28.6 & 11.7 & 11.3 & 5.1 & 1.2 & 0.0 & 0.0 & 0.0 & 3.1 & 0.1 & 1.1 & 2.1 \\
\hline Serbia, Ovča & [84] & 2.0 & 28.2 & 11.7 & 1.8 & 2.2 & 0.9 & 0.0 & 0.0 & 0.5 & 7.9 & 0.4 & 0.1 & 1.7 \\
\hline Lithuania1 & [85] & 1.7 & 14.2 & 8.0 & 4.9 & 3.8 & 3.9 & 1.6 & 0.0 & 0.0 & 7.1 & 1.8 & 0.0 & 4.1 \\
\hline Lithuania2 & [85] & 2.2 & 14.0 & 7.8 & 3.8 & 4.9 & 1.8 & 1.2 & 0.0 & 0.0 & 5.3 & 0.8 & 0.0 & 2.8 \\
\hline Lithuania3 & [85] & 0.9 & 15.5 & 10.1 & 2.8 & 2.3 & 3.6 & 2.0 & 0.0 & 0.0 & 5.7 & 1.0 & 0.0 & 3.4 \\
\hline Lithuania4 & [85] & 2.1 & 15.2 & 11.8 & 2.8 & 3.9 & 1.7 & 2.1 & 0.0 & 0.0 & 5.6 & 0.9 & 0.0 & 2.7 \\
\hline Lithuania5 & [85] & 7.0 & 10.2 & 7.7 & 0.6 & 0.4 & 0.9 & 1.2 & 0.0 & 0.0 & 7.5 & 2.8 & 0.0 & 4.8 \\
\hline Lithuania6 & [85] & 7.2 & 17.2 & 9.3 & 4.7 & 2.5 & 5.3 & 0.6 & 0.0 & 0.0 & 3.7 & 0.7 & 0.0 & 0.0 \\
\hline Lithuania7 & [85] & 6.5 & 15.6 & 6.7 & 6.6 & 4.3 & 7.6 & 0.9 & 0.0 & 0.0 & 3.0 & 1.0 & 0.0 & 0.0 \\
\hline Lithuania8 & [85] & 13.0 & 13.6 & 9.4 & 3.6 & 1.5 & 5.9 & 1.2 & 0.0 & 0.0 & 3.7 & 1.1 & 0.0 & 0.0 \\
\hline Lithuania16 & [85] & 1.9 & 12.3 & 6.4 & 3.5 & 7.6 & 2.2 & 3.3 & 0.0 & 0.0 & 5.8 & 1.4 & 0.0 & 0.0 \\
\hline Lithuania17 & [85] & 1.5 & 12.1 & 3.1 & 3.6 & 8.0 & 2.0 & 3.6 & 0.0 & 0.0 & 1.9 & 0.8 & 0.0 & 0.0 \\
\hline Lithuania18 & [85] & 7.1 & 9.1 & 6.4 & 1.8 & 2.7 & 2.0 & 2.2 & 0.0 & 0.0 & 3.2 & 1.5 & 0.0 & 0.9 \\
\hline Lithuania19 & [85] & 5.4 & 7.0 & 4.5 & 0.9 & 3.7 & 1.6 & 1.6 & 0.0 & 0.0 & 5.5 & 1.9 & 0.0 & 0.0 \\
\hline Serbia, Sokobanja4 & {$[84]$} & 6.1 & 8.1 & 12.9 & 3.0 & 6.6 & 3.5 & 0.0 & 0.1 & 0.1 & 1.9 & 0.5 & 3.9 & 0.0 \\
\hline Serbia, Sokobanja 5 & [84] & 2.6 & 12.6 & 14.5 & 3.8 & 0.9 & 0.5 & 0.0 & 0.0 & 0.0 & 8.3 & 0.3 & 0.0 & 2.2 \\
\hline Serbia, Aleksinac1 & [84] & 8.0 & 3.6 & 10.4 & 1.1 & 0.0 & 3.0 & 0.0 & 0.0 & 0.3 & 4.0 & 0.4 & 7.6 & 0.0 \\
\hline Serbia, Aleksinac2 & [84] & 12.2 & 2.9 & 17.3 & 1.1 & 1.3 & 2.6 & 0.0 & 0.0 & 0.0 & 2.5 & 0.3 & 15.5 & 0.0 \\
\hline Serbia, Vojska & [84] & 10.2 & 0.8 & 14.3 & 1.1 & 1.7 & 6.5 & 0.0 & 0.0 & 0.3 & 0.8 & 0.2 & 12.5 & 0.0 \\
\hline Serbia, Velika Plana & [84] & 6.1 & 3.9 & 13.6 & 1.4 & 13.5 & 1.9 & 0.0 & 0.0 & 0.8 & 3.3 & 0.4 & 4.8 & 0.9 \\
\hline
\end{tabular}


Table 6. Cont.

\begin{tabular}{|c|c|c|c|c|c|c|c|c|c|c|c|c|c|c|}
\hline Locality & Ref. & & 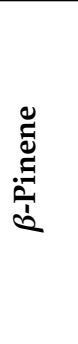 & 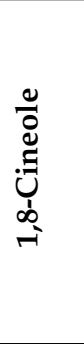 & Ũ & D' & 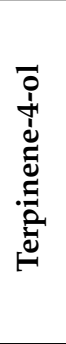 & 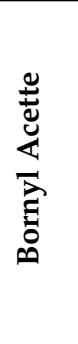 & 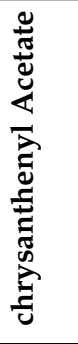 & 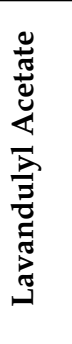 & 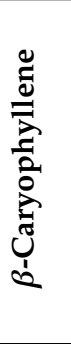 & 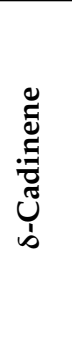 & $\begin{array}{l}\text { 올 } \\
\frac{0}{\overrightarrow{0}}\end{array}$ & 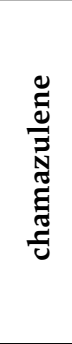 \\
\hline Serbia, Begaljica & [84] & 6.9 & 19.6 & 26.6 & 2.6 & 9.1 & 1.2 & 0.0 & 0.0 & 0.0 & 7.4 & 0.2 & 0.1 & 0.0 \\
\hline Italy, Cagliary & [86] & 14.6 & 1.1 & 17.2 & 1.6 & 1.6 & 5.7 & 0.0 & 0.0 & 0.0 & 2.3 & 0.0 & 0.0 & 0.1 \\
\hline Iran, Chaharbagh & [87] & 3.2 & 2.1 & 18.6 & 13.9 & 9.4 & 1.2 & 1.3 & 0.0 & 0.0 & 2.1 & 0.0 & 0.0 & 2.3 \\
\hline Turkey, Sivaz & [88] & 2.8 & 4.2 & 24.6 & 16.7 & 4.0 & 2.8 & 0.1 & 0.0 & 0.0 & 0.4 & 0.0 & 0.0 & 0.0 \\
\hline Lithuania9 & [85] & 4.5 & 7.3 & 9.6 & 5.9 & 2.4 & 1.7 & 1.6 & 0.0 & 0.0 & 2.6 & 1.3 & 0.0 & 1.2 \\
\hline Lithuania10 & [85] & 3.7 & 9.5 & 9.9 & 2.0 & 2.0 & 1.9 & 4.7 & 0.0 & 0.0 & 2.6 & 0.6 & 0.0 & 0.0 \\
\hline Lithuania11 & [85] & 2.5 & 6.6 & 8.8 & 2.4 & 2.5 & 1.3 & 3.7 & 0.0 & 0.0 & 6.5 & 1.0 & 0.0 & 0.8 \\
\hline Serbia, Rtanj & TS & 2.8 & 1.5 & 5.6 & 9.8 & 1.6 & 6.3 & 0.4 & 0.4 & 0.0 & 4.7 & 0.0 & 0.7 & 0.1 \\
\hline France, Toulouse & [89] & 6.7 & 3.4 & 4.0 & 12.8 & 1.8 & 4.7 & 1.2 & 0.0 & 0.0 & 1.7 & 1.2 & 1.6 & 0.0 \\
\hline Lithuania15 & [85] & 3.6 & 4.5 & 8.8 & 13.1 & 12.8 & 2.2 & 0.7 & 0.0 & 0.0 & 0.7 & 0.3 & 0.0 & 0.0 \\
\hline Serbia, Sokobanja3 & [84] & 1.9 & 15.4 & 14.3 & 0.9 & 20.2 & 1.2 & 0.0 & 0.0 & 0.1 & 2.5 & 0.2 & 8.4 & 0.0 \\
\hline Lithuania12 & [85] & 4.3 & 6.5 & 9.5 & 4.1 & 11.5 & 2.6 & 1.1 & 0.0 & 0.0 & 3.5 & 0.6 & 0.0 & 0.7 \\
\hline Lithuania13 & [85] & 1.5 & 4.0 & 5.3 & 7.2 & 13.1 & 1.9 & 1.9 & 0.0 & 0.0 & 1.9 & 0.6 & 0.0 & 1.3 \\
\hline Lithuania14 & [85] & 3.1 & 12.6 & 12.5 & 7.2 & 13.2 & 4.5 & 0.5 & 0.0 & 0.0 & 2.5 & 0.5 & 0.0 & 0.0 \\
\hline Serbia, Secanj & [90] & 2.8 & 3.2 & 0.1 & 2.0 & 1.4 & 0.0 & 0.0 & 21.3 & 0.9 & 9.5 & 0.0 & 0.0 & 1.5 \\
\hline Serbia, Ćuprija & {$[84]$} & 5.6 & 5.6 & 2.1 & 9.4 & 3.1 & 1.1 & 0.0 & 0.0 & 11.0 & 5.3 & 0.4 & 1.1 & 0.2 \\
\hline Serbia, Aradac & [90] & 3.6 & 4.1 & 3.5 & 1.0 & 5.3 & 0.0 & 0.0 & 6.8 & 14.9 & 7.6 & 0.5 & 0.0 & 13.9 \\
\hline Serbia, Suvobor & [84] & 1.2 & 10.1 & 2.0 & 0.2 & 0.0 & 0.8 & 0.0 & 0.0 & 0.0 & 12.1 & 0.8 & 0.0 & 3.4 \\
\hline Turkey, Elazig & [91] & 2.7 & 0.3 & 2.3 & 0.0 & 0.0 & 0.0 & 0.0 & 0.0 & 2.3 & 4.9 & 19.0 & 0.6 & 0.0 \\
\hline Serbia, Maljen & [84] & 8.9 & 22.2 & 1.9 & 0.1 & 0.1 & 1.3 & 0.0 & 0.0 & 1.6 & 13.9 & 0.6 & 0.0 & 29.1 \\
\hline Estonia & [84] & 6.6 & 17.6 & 7.6 & 0.3 & 0.4 & 0.2 & 0.4 & 0.0 & 0.0 & 9.8 & 1.2 & 0.0 & 30.7 \\
\hline UK & [92] & 4.5 & 11.5 & 4.9 & 4.3 & 1.8 & 0.4 & 0.7 & 0.2 & 0.0 & 6.6 & 1.1 & 0.0 & 25.8 \\
\hline Latvia & [92] & 4.0 & 12.0 & 4.7 & 1.7 & 1.5 & 0.2 & 1.5 & 0.1 & 0.0 & 6.7 & 1.3 & 0.0 & 26.8 \\
\hline Norway & [92] & 2.7 & 9.3 & 2.9 & 1.1 & 0.9 & 0.3 & 1.1 & 0.0 & 0.0 & 6.3 & 1.3 & 0.0 & 40.2 \\
\hline Lithuania20 & [85] & 3.2 & 15.1 & 6.4 & 0.5 & 1.2 & 2.3 & 0.0 & 0.0 & 0.0 & 8.0 & 2.1 & 0.0 & 20.1 \\
\hline Serbia, Divčibare2 & [84] & 3.1 & 10.2 & 18.1 & 0.9 & 0.8 & 0.8 & 0.0 & 0.0 & 0.0 & 5.1 & 0.3 & 0.0 & 22.2 \\
\hline Ukraine & [92] & 2.0 & 5.9 & 6.0 & 1.2 & 1.2 & 1.7 & 0.7 & 0.1 & 0.0 & 4.3 & 1.7 & 0.0 & 23.7 \\
\hline Germany & [92] & 0.0 & 0.1 & 0.0 & 0.1 & 1.4 & 1.2 & 7.3 & 0.0 & 0.0 & 2.5 & 1.2 & 0.0 & 44.3 \\
\hline Austria & [92] & 4.9 & 7.7 & 2.5 & 0.9 & 1.7 & 3.1 & 15.8 & 0.0 & 0.0 & 3.6 & 0.5 & 0.0 & 15.7 \\
\hline Serbia, Padej & [84] & 6.3 & 0.9 & 1.7 & 0.3 & 0.0 & 0.6 & 0.0 & 0.8 & 18.1 & 3.2 & 0.3 & 0.0 & 23.1 \\
\hline Serbia, Divčibare1 & {$[84]$} & 0.6 & 11.0 & 4.5 & 0.3 & 0.0 & 0.4 & 0.0 & 0.0 & 8.2 & 15.8 & 0.2 & 0.1 & 18.3 \\
\hline Serbia, Slano Kopovo & [90] & 5.3 & 8.9 & 5.3 & 1.5 & 1.0 & 0.0 & 0.0 & 5.8 & 0.9 & 9.0 & 0.0 & 0.0 & 15.8 \\
\hline Czech & [92] & 0.0 & 0.0 & 0.8 & 0.7 & 0.6 & 0.5 & 1.2 & 0.0 & 0.0 & 9.7 & 1.9 & 0.0 & 25.8 \\
\hline
\end{tabular}




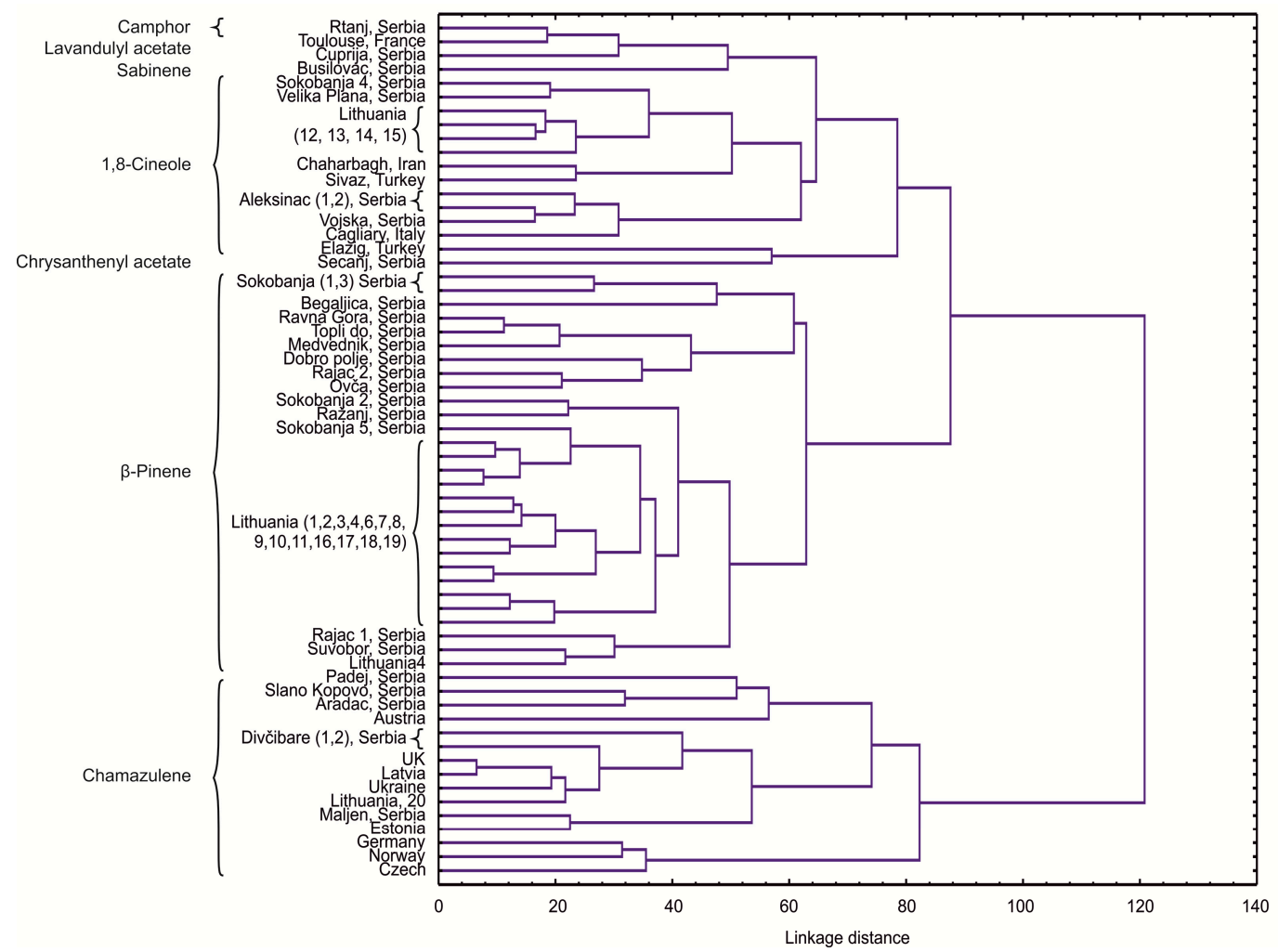

(a)

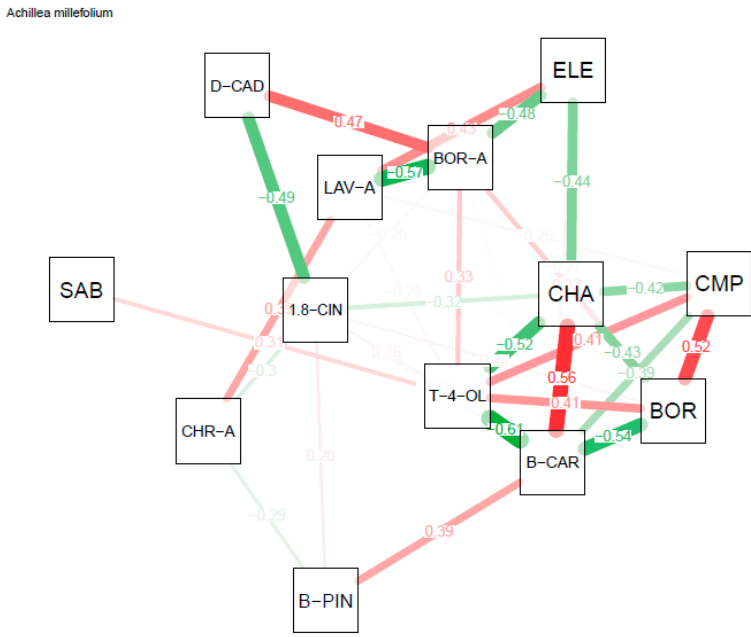

(b)

Figure 4. (a) Dendogram of the A. millefolium EO constituents from this study and from literature reports (the samples are marked according to Table 6); (b) correlation network based on A. millefolium EO constituents (SAB — sabinene; B-PIN— $\beta$-pinene; $\mathrm{CMP}$ — camphor; 1,8 -CIN—1,8-cineole; BOR—borneole; T-4-OL—terpinene-4-ol; BOR-A—bornyl acetate; CHR-A—chrysanthenyl acetate; LAV-A-lavandulyl acetate; $\mathrm{CHA}$-chamazulene; B-CAR— $\beta$-caryophyllene; D-CAD— $\delta$-cadinene; ELE—elemol.

According to the major compound of the EO, this $A$. millefolium from Rtanj can be classified as a camphor chemotype. Essential oil of $A$. millefolium from France was also identified as a camphor chemotype (12.8\% camphor in EO) [89]. However, in Lithuania, six different chemotypes of A. millefolium were found: $\beta$-pinene, 1,8-cineole, borneol, camphor, nerolidol and chamazulene chemotypes [85]. In Poland, three chemotypes were found based on the determination of the variability between 20 yarrow populations according to content of the most dominant compounds identified in the EO: 
$\beta$-pinene, $\beta$-pinene + chamazulene and 1,8-cineole chemotype [93]. Variations in the essential oil content and composition in commercial samples of yarrow from different European countries were analyzed, and five chemotypes were determined: chamazulene, chamazulene + bornyl acetate, chamazulene + $\beta$-pinene + trans- $\beta$-caryophyllene, sabinene $+1,8$-cineole and $\beta$-pinene $+\alpha$-terpinyl acetate [92]

The analysis of 28 populations of $A$. millefolium collected from Serbian sites showed that the most dominant compounds were $\beta$-pinene, sabinene, 1,8-cineole, borneol, trans-caryophyllene, lavandulyl acetate and chamazulene [84]. Furthermore, investigations in Serbia showed that the high percentage of oxygenated monoterpenes and absence of azulene in the obtained EO proved that this population was octaploid, whereas the chamazulene chemotype was in the tetraploid population [94]. In addition, collections from saline habitats in Serbia identified three chemotypes: chamazulene + trans-caryophyllene $+\beta$-pinene $(15.84+8.98+8.89 \%$, respectively $)$, lavandulyl acetate + chamazulene + trans-caryophyllene $(14.88+13.89+7.57 \%$, respectively) and trans-chrysanthenyl acetate + trans-caryophyllene + germacrene $\mathrm{D}(21.33+9.53+7.07 \%$, respectively $)$ [90].

Taking into account the chemical content range, a correlation network based on components from EO of this species is shown in Figure $4 \mathrm{~b}$. The strongest negative multiple correlations were found between $\beta$-caryophyllene and terpinene-4-ol $(\mathrm{r}=-0.61 ; p<0.01)$ and the strongest positive correlations were between $\beta$-caryophyllene and chamazulene $(\mathrm{r}=0.56 ; p<0.01)$.

The A. millefolium EO showed antimicrobial activity in vitro against Streptococcus pneumoniae, Clostridium perfringens, C. albicans, C. krusei, Mycobacterium smegmatis and Acinetobacter lwoffii [88]. Additionally, in vitro antibacterial activity against nine Gram positive and negative bacteria (S. epidermidis, S. aureus, B. cereus, E. faecalis, E. coli, P. aeruginosa, K. pneumoniae, S. typhimurium and Shigella dysenteria) demonstrated that $A$. millefolium EO can potentially be used for controlling certain bacteria that cause many infectious diseases, but its effectiveness varied in different regions because of the differences in EO composition [87].

\subsection{Achillea clypeolata}

The main compounds in the A. clypeolata EO from Mt. Rtanj (collected in July 1996) were 1,8-cineole (38.6\%) and camphor (19.9\%) [95]. The concentration of 1,8 cineole in A. clypeolata in this study was a bit higher. This variation in the chemical composition could be attributed to the weather conditions during the year, collection time, population and exposition. Because this is an endemic species in the Balkan region, there were very few studies on its EO composition. A. clypeolata of Serbian origin contained 1,8-cineole as the dominant compound [95,96], and 1,8-cineole as a potential chemotype of this species was confirmed by this study. However, A. clypeolata grown in a botanical garden in Italy could be characterized as $\beta$-pinene chemotype [97].

The differentiation between A. clypeolata EO samples (Table 7) was performed using HCA analysis (Figure 5a). Only two clusters (potential chemotypes) were established, including 1,8-cineole and $\beta$-pinene chemotype.

Taking into account the chemical content range, a correlation network based on components from $\mathrm{EO}$ of this species is shown in Figure $5 \mathrm{~b}$. There were strong negative correlations between terpinen-4-ol and $\beta$-pinene as well as $\beta$-pinene and borneol. Positive correlations are noted between terpinen-4-ol and borneol, as well as multiple correlation between allo-aromadendrene, epi- $\alpha$-cadinol and $\alpha$-cadinol.

A. clypeolata has not been investigated thoroughly yet, with the exception of its antioxidant and antimicrobial properties [96,98]. In this study, the $\mathrm{EO}$ of $A$. clypeolata exhibited the strongest activity against K. pneumoniae and P. aeruginosa, even stronger than the antibiotic Ampicilin, which was used as the standard for comparison. A. clypeolata EO showed lower activity against Gram-positive S. aureus, but still stronger than Ampicilin, while E. coli was the most resistant to the oil [96]. Indeed, previous study on Cinnamomum longepaniculatum leaf EO has shown that its compounds have excellent antibacterial activities, and the antibacterial mechanism of 1,8-cineole against $E$. coli and S. aureus might be attributed to its hydrophobicity [99]. 
Table 7. A. clypeolata EO constituents from this study and others reported previously in the literature.

\begin{tabular}{|c|c|c|c|c|c|c|c|c|c|c|c|c|c|c|}
\hline Locality & Ref. & 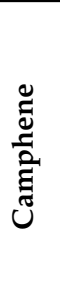 & $\begin{array}{c}\stackrel{0}{0} \\
\stackrel{\Xi}{0} \\
\tilde{\Xi}\end{array}$ & 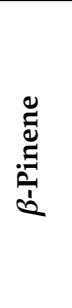 & 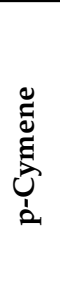 & 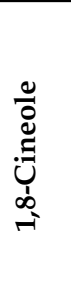 & 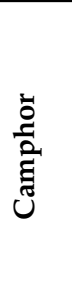 & 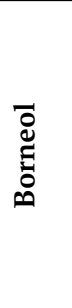 & 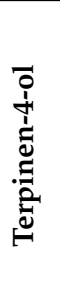 & 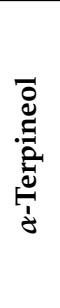 & 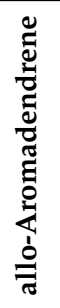 & 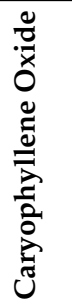 & 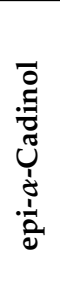 & 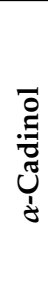 \\
\hline Serbia, Rtanj & TS & 2.3 & 0.3 & 1.4 & 3.1 & 45.1 & 18.2 & 2.7 & 2.8 & 2.4 & 1.3 & 3.2 & 2.4 & 1.5 \\
\hline Serbia, Rtanj & [95] & 1.9 & 0.0 & 0.8 & 3.6 & 38.6 & 19.9 & 3.6 & 6.5 & 5.3 & 0.4 & 1.7 & 1.9 & 1.1 \\
\hline Serbia, Rudina & [96] & 0.0 & 0.0 & 0.0 & 0.0 & 16.0 & 9.2 & 11.9 & 8.8 & 2.4 & 0.0 & 11.5 & 0.0 & 0.0 \\
\hline Italy, Turin & [97] & 1.0 & 9.2 & 23.7 & 1.8 & 10.1 & 2.2 & 1.2 & 2.6 & 2.6 & 0.0 & 0.8 & 0.0 & 0.0 \\
\hline
\end{tabular}

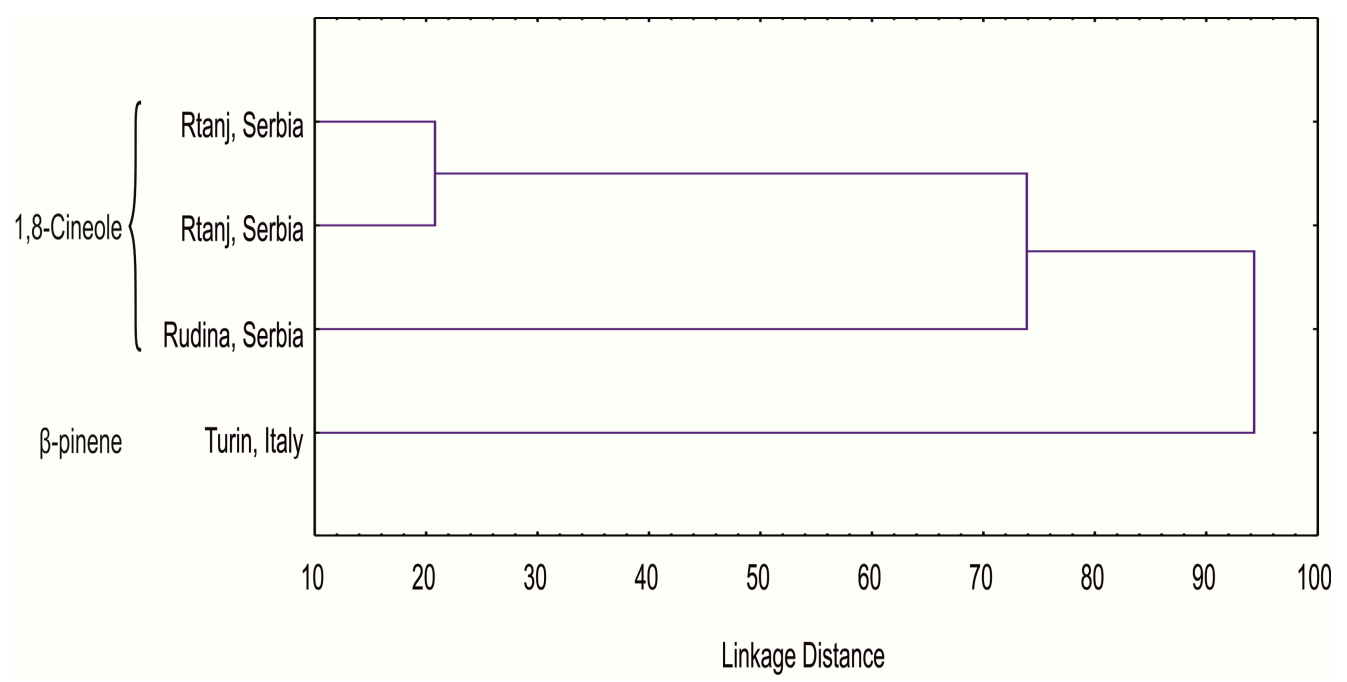

(a)
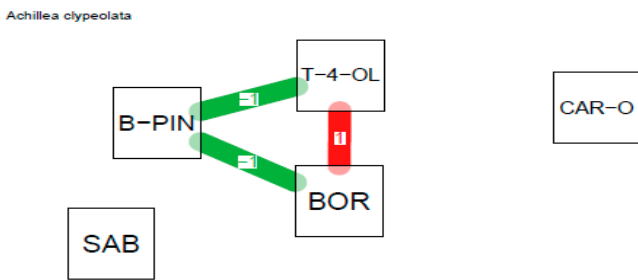

A-TER
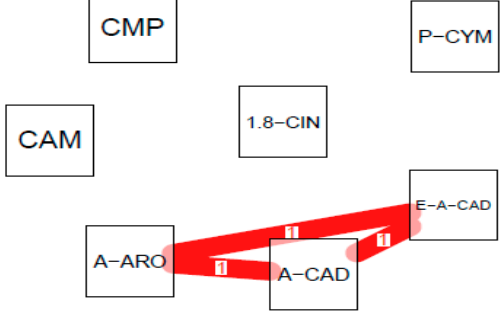

(b)

Figure 5. (a) Dendogram of the EO constituents of $A$. clypeolata from this study and from literature reports (the samples are marked according to Table 7); (b) correlation network based on $A$. clypeolata EO constituents (CAM — camphene; $\mathrm{SAB}$ — sabinene; B-PIN— $\beta$-pinene; P-CYM- $\mathrm{p}$-cymene; $1,8-\mathrm{CIN}$-1,8-cineole; $\mathrm{CMP}$ —camphor; BOR—borneol; T-4-OL—terpinene-4-ol; A-TER— $\alpha$-terpineol; A-ARO—allo-aromadendrene; CAR-O—caryophyllene oxide; E-A-CAD—epi- $\alpha$-cadinol; A-CAD— $\alpha$-cadinol. 


\section{Material and Methods}

\subsection{Plant Material}

The plant species were collected at full flowering stage in July 2018. Satureja kitaibelii Wierzb. ex Heuff., Origanum vulgare L. ssp. vulgare, Achillea millefolium L. sensu lato and A. clypeolata Sibth. \& Sm were collected on Mt. Rtanj and Thymus serpyllum L. was collected in the village of Sesalac. The aboveground parts were harvested by cutting them manually at around $2-3 \mathrm{~cm}$ above the soil surface and were then dried in a shady well-aerated place to a constant mass.

Voucher specimens were identified by Dr. Milica Rat and deposited at the Herbarium BUNS, the University of Novi Sad, Faculty of Sciences, Department of Biology and Ecology as S. kitaibelii Wierzb. ex Heuff. (2-1442), T. serpyllum L. (2-1444), O. vulgare L. ssp. vulgare (2-1450), A. millefolium L. sensu lato (2-1449) and A. clypeolata Sibth. \& Sm (2-1448).

\subsection{Essential Oil (EO) Extraction}

Clevenger apparatus was used to extract the essential oil from the air-dried aboveground parts of each sample in three replications. According to the European Pharmacopoeia, $30 \mathrm{~g}$ each of the drug plants from the Lamiaceae family (S. kitaibelii, T. serpyllum and O. vulgare ssp. vulgare) and $400 \mathrm{~mL}$ of water and $20 \mathrm{~g}$ each of cut drug from the Asteraceae family (A. millefolium and A. clypeolata) and $500 \mathrm{~mL}$ of water were placed separately in $1000 \mathrm{~mL}$ round bottom flasks and distilled for $3 \mathrm{~h}$ [100].

The EO yield as an average of three replications was S. kitaibelii $(0.09 \%)$, T. serpyllum $(0.26 \%)$, O. vulgare ssp. vulgare (0.12\%), A. millefolium (0.16\%) and A. clypeolata (0.04\%). The obtained EOs were dried over anhydrous $\mathrm{NaSO}_{4}$ and stored in the dark at $4{ }^{\circ} \mathrm{C}$ for further analysis.

\subsection{Essential Oil (EO) Analysis}

Gas chromatography-mass spectrometry (GC-MS) was performed using an HP 5890 coupled with an HP 5973 MSD fitted with an HP-5MS capillary column (conditions in detail described by Aćimović et al. [101]). The components were identified based on their linear retention index relative to C8-C32 n-alkanes, in comparison with data reported in the literature (Wiley and NIST databases). The percentage (relative) of the identified compounds was computed from the GC peak area.

\subsection{Association among Chemical Compounds}

The association of the chemical compounds of EOs was estimated using the Spearman non-parametric correlation coefficient. In order to further analyze and represent the associations among the chemical compounds, a correlation network graph [102] was used. In this type of graph, the chemical compound variables are represented by the nodes, which are connected by the edge whose width is directly proportional to the strength of the correlation coefficient. The correlation network graph is easier to interpret than a numerical correlation matrix, and the pattern of the correlations, i.e., the clusters of the correlated variables, can be visually identified [103].

A data set, composed of 16 S. kitaibelii, 54 T. serpyllum, 53 O. vulgare ssp. vulgare, 63 A. millefolium and $4 \mathrm{~A}$. clypeolata samples and 13 variables (the main EO constituents), were depicted using a correlation network graph for visualization correlations between chemical compounds from EOs (Spearman's rank order correlation coefficient, r). Non-significant correlations were removed from the correlation network graph.

\subsection{Antibacterial Activity}

The antimicrobial activities of the tested EOs were investigated using American Type Culture Collection test strains of Escherichia coli (ATCC 8739) and Staphylococcus aureus (ATCC 25923). Strains were cultured on Tryptone Soya Agar (TSA) and incubated at $37^{\circ} \mathrm{C}$ for $24 \mathrm{~h}$. Isolated colonies were picked and transferred to $5 \mathrm{~mL}$ of Tryptone Soy Broth (TSB) and incubated at $37^{\circ} \mathrm{C}$ for $18 \mathrm{~h}$. The density 
of the suspensions used for tests was adjusted to $0.5 \mathrm{Mc}$ Farland units $\left(\sim 1-2 \times 10^{8} \mathrm{CFU} / \mathrm{mL}\right)$ using a densitometer DEN-1 and standard plate counts. Efficacy of EOs on microorganisms was determined according to the CLSI (2018) [104] with slight modifications [105].

Tested EO was dissolved with sterile water supplemented with $0.05 \%$ Tween 80 and added to 96-well microtiter plates at concentrations from 200 to $0.781 \mu \mathrm{L} / \mathrm{mL}$ (the final concentration in microtiter plates was from 20 to $0.078 \mu \mathrm{L} / \mathrm{mL}$ ). The $160 \mu \mathrm{L}$ in Mueller-Hinton Broth (MHB) were also added to each well, and in the end, $20 \mu \mathrm{L}$ of overnight bacterial cultures suspensions were inoculated. Plates were incubated at $37^{\circ} \mathrm{C}$ for $24 \mathrm{~h}$. After incubation, $20 \mu \mathrm{L}$ of the resazurin solution $(0.01 \%)$ were added to each well, and the plates were further incubated at $37^{\circ} \mathrm{C}$ for $24 \mathrm{~h}$ in darkness. A change of color from blue (oxidized-resazurin remained unchanged) to pink (reduced) indicated the growth of bacteria.

Referring to the results of the minimal inhibitory concentration (MIC), the wells showing a complete absence of growth were identified and $100 \mu \mathrm{L}$ of the solutions from each well were transferred to plate count agar plates (PCA) and incubated at $37^{\circ} \mathrm{C}$ for $24 \mathrm{~h}$. The minimal bactericidal concentration (MBC) was defined as the lowest concentration of the EOs at which $99.9 \%$ of the inoculated microorganisms were killed.

\subsection{Statistical Analysis}

Experimental results were expressed as mean \pm standard deviation, with 10 repetitions for microbiological analyses. Analysis of variance (ANOVA) for comparison of sample means and a post-hoc Tukey's HSD test was used to analyze variations in observed parameters among the samples.

Hierarchical cluster analysis (HCA) was used to evaluate intra- and interpopulation variability and differentiation of EO constituents of S. kitaibelii, T. serpyllum, O. vulgare ssp. vulgare, A. millefolium and A. clypeolata in samples collected in different locations and/or taken from literature reports. Data was analyzed using StatSoft Statistica 12.

\section{Conclusions}

This is the first study to characterize EO composition and antimicrobial activity of the five medicinal species from Eastern Serbia against comprehensive literature data. This study demonstrated that $S$. kitaibelii plants from Mt. Rtanj belonged to the $p$-cymene chemotype, T. serpyllum plants belonged to the geraniol chemotype, while the O. vulgare ssp. vulgare plants belonged to the germacrene $\mathrm{D}$ chemotype. The A. millefolium were recorded as a multicomponent chemotype, while A. clypeolata belonged to 1,8-cineole (45.1\%) chemotype. Furthermore, the S. kitaibelii EO was demonstrated to be a promising agent against $S$. aureus and E. coli bacterial strains. The chemical composition of studied EOs particularly focuses on the main EO constituents, which are assumed to be responsible for the observed antibacterial activity. Further investigations will be focused on studying major constituents of these EOs to evaluate concentrations of the components that could be responsible for the observed effect. Additional tests with larger numbers of bacteria regarding the synergic potential of EOs will be implemented in our future investigations.

Author Contributions: Conceptualization, M.A. and V.D.Z.; methodology, M.A.; software, M.Z.; validation, J.S.J. and M.C.; formal analysis, J.S.J.; investigation, M.C.; resources, M.A.; data curation, M.Z. and L.P.; writing_-original draft preparation, M.A. and I.Č.; writing—review and editing, V.D.Z. and I.Č.; visualization, M.Z. and L.P.; supervision, V.D.Z.; project administration, V.D.Z.; funding acquisition, V.D.Z. All authors have read and agreed to the published version of the manuscript.

Funding: This research received no external funding.

Acknowledgments: The authors gratefully acknowledge Adonis Sokobanja for their administrative and technical support.

Conflicts of Interest: The authors declare no conflict of interest. 


\section{References}

1. Carović-Stanko, K.; Petek, M.; Grdiša, M.; Pintar, J.; Bedeković, D.; Herak Ćustić, M.; Satovic, Z. Medicinal plants of the family Lamiaceae as functional foods-A review. Czech J. Food Sci. 2016, 34, 377-390. [CrossRef]

2. Bessada, S.M.F.; Barreira, J.C.M.; Oliveira, M.B.P.P. Asteraceae species with most prominent bioactivity and their potential applications: A review. Ind. Crops Prod. 2015, 76, 604-615. [CrossRef]

3. Cheminal, A.; Kokkoris, I.P.; Strid, A.; Dimopoulos, P. Medicinal and aromatic Lamiaceae plants in Greece: Linking diversity and distribution patterns with ecosystem services. Forests 2020, 11, 661. [CrossRef]

4. Akinyede, K.A.; Ekpo, O.E.; Oguntibeju, O.O. Ethnopharmacology, therapeutic properties and nutritional potentials of Carpobrotus edulis: A comprehensive review. Sci. Pharm. 2020, 88, 39. [CrossRef]

5. Nyakudya, T.T.; Tshabalala, T.; Dangarembizi, R.; Erlwanger, K.; Ndhlala, A.R. The potential therapeutic value of medicinal plants in the management of metabolic disorders. Molecules 2020, 25, 2669. [CrossRef] [PubMed]

6. Tavares, W.R.; Barreto, M.C.; Seca, A.M.L. Uncharted source of medicinal products: The case of the Hedychium genus. Medicines 2020, 7, 23. [CrossRef]

7. Ahmed, S.M.; Sundby, J.; Aragaw, Y.A.; Abebe, F. Self-medication and safety profile of medicines used among pregnant women in a Tertiary Teaching Hospital in Jimma, Ethiopia: A cross-sectional study. Int. J. Environ. Res. Public Health 2020, 17, 3993. [CrossRef]

8. Singh, B.; Singh, B.; Kishor, A.; Singh, S.; Bhat, M.N.; Surmal, O.; Musarella, C.M. Exploring plant-based ethnomedicine and quantitative ethnopharmacology: Medicinal plants utilized by the population of Jasrota Hill in Western Himalaya. Sustainability 2020, 12, 7526. [CrossRef]

9. Salmerón-Manzano, E.; Garrido-Cardenas, A.J.; Manzano-Agugliaro, F. Worldwide research trends on medicinal plants. Int. J. Environ. Res. Public Health 2020, 17, 3376. [CrossRef]

10. Ebadollahi, A.; Ziaee, M.; Palla, F. Essential oils extracted from different species of the Lamiaceae plant family as prospective bioagents against several detrimental pests. Molecules 2020, 25, 1556. [CrossRef]

11. Durán-Lara, E.F.; Valderrama, A.; Marican, A. Natural organic compounds for application in organic farming. Agriculture 2020, 10, 41. [CrossRef]

12. Raveau, R.; Fontaine, J.; Sahraoui, A.L.H. Essential oils as potential alternative biocontrol products against plant pathogens and weeds: A review. Foods 2020, 9, 365. [CrossRef] [PubMed]

13. Marković, M.; Stankov Jovanović, V.; Smiljić, M. Medicinal flora of the Vidlič Mountain in Serbia. Univ. Thought Publ. Nat. Sci. 2019, 9, 17-26. [CrossRef]

14. Gostuški, R. Lečenje Lekovitim Biljem; IRO Narodna Knjiga: Belgrade, Serbia, 1979.

15. Tucakov, J. Healing with Plants_Phytotherapy; Rad: Belgrade, Serbia, 2006; ISBN 86-09-00431-7.

16. Čančarević, A.; Bugarski, B.; Šavikin, K.; Zdunić, G. Biological activity and ethnomedicinal use of Thymus vulgaris and Thymus serpyllum. Lek. Sirovine 2013, 33, 3-17.

17. Stanojković, T.; Kolundžija, B.; Ćirić, A.; Soković, M.; Nikolić, D.; Kundaković, T. Cytotoxicity and antimicrobial activity of Satureja kitaibelii Wierzb. ex Heuff (Lamiaceae). Dig. J. Nanomater. Biostruct. 2013, 8, 845-854.

18. Dodoš, T.; Rajčević, N.; Janaćković, P.; Vujisić, L.; Marin, P. Essential oil profile in relation to geographic origin and plant organ of Satureja kitaibelii Wierzb. ex Heuff. Ind. Crops Prod. 2019, 139, 111549. [CrossRef]

19. Prakapaite, R.; Saab, F.; Planciuniene, R.; Petraitis, V.; Walsh, T.J.; Petraitiene, R.; Semoskaite, R.; Baneviciene, R.; Kalediene, L.; Kavaliauskas, P. Molecular characterization of uropathogenic Escherichia coli reveals emergence of drug resistant O15, O22 and O25 Serogroups. Medicina 2019, 55, 733. [CrossRef]

20. Hrbacek, J.; Cermak, P.; Zachoval, R. Current antibiotic resistance trends of uropathogens in Central Europe: Survey from a Tertiary hospital urology department 2011-2019. Antibiotics 2020, 9, 630. [CrossRef]

21. Sokhn, E.S.; Salami, A.; Roz, A.E.; Salloum, L.; Bahmad, H.F.; Ghssein, G. Antimicrobial susceptibilities and laboratory profiles of Escherichia coli, Klebsiella pneumoniae, and Proteus mirabilis isolates as agents of urinary tract infection in Lebanon: Paving the way for better diagnostics. Med. Sci. 2020, 8, 32. [CrossRef]

22. Yossa, N.; Patel, J.; Millner, P.; Lo, M. Essential oils reduce Escherichia coli O157:H7 and Salmonella on spinach leaves. J. Food Prot. 2012, 75, 488-496. [CrossRef]

23. Paswan, R.; Park, Y.W. Survivability of Salmonella and Escherichia coli O157:H7 pathogens and food safety concerns on commercial powder milk products. Dairy 2020, 1, 189-201. [CrossRef] 
24. Syahrul, F.; Wahyuni, C.U.; Notobroto, H.B.; Wasito, E.B.; Adi, A.C.; Dwirahmadi, F. Transmission media of foodborne diseases as an index prediction of diarrheagenic Escherichia coli: Study at elementary school, Surabaya, Indonesia. Int. J. Environ. Res. Public Health 2020, 17, 8227. [CrossRef] [PubMed]

25. Gallina, S.; Bianchi, D.M.; Bellio, A.; Nogarol, C.; Macori, G.; Zaccaria, T.; Biorci, F.; Carraro, E.; Decastelli, L. Staphylococcal poisoning foodborne outbreak: Epidemiological investigation and strain genotyping. J. Food Prot. 2013, 76, 2093-2098. [CrossRef]

26. Abril, A.G.; Villa, T.G.; Barros-Velázquez, J.; Cañas, B.; Sánchez-Pérez, A.; Calo-Mata, P.; Carrera, M. Staphylococcus aureus exotoxins and their detection in the dairy industry and mastitis. Toxins 2020, 12, 537. [CrossRef] [PubMed]

27. Higaki, S.; Kitagawa, T.; Kagoura, M.; Morohashi, M.; Yamagishi, T. Predominant Staphylococcus aureus isolated from various skin diseases. J. Int. Med. Res. 2000, 28, 187-190. [CrossRef] [PubMed]

28. Bouvet, C.; Gjoni, S.; Zenelaj, B.; Lipsky, B.A.; Hakko, E.; Uçkay, I. Staphylococcus aureus soft tissue infection may increase the risk of subsequent staphylococcal soft tissue infections. Int. J. Infect. Dis. 2017, 60, 44-48. [CrossRef] [PubMed]

29. Principi, N.; Argentiero, A.; Neglia, C.; Gramegna, A.; Esposito, S. New antibiotics for the treatment of acute bacterial skin and soft tissue infections in pediatrics. Pharmaceuticals 2020, 13, 333. [CrossRef] [PubMed]

30. Appelbaum, P.C. Microbiology of antibiotic resistance in Staphylococcus aureus. Clin. Infect. Dis. 2007, 15, S165-S170. [CrossRef] [PubMed]

31. Gregova, G.; Kmetova, M.; Kmet, V.; Venglovsky, J.; Feher, A. Antibiotic resistance of Escherichia coli isolated from a poultry slaughterhouse. Ann. Agric. Environ. Med. 2012, 19, 75-77.

32. Musa, L.; Proietti, P.C.; Branciari, R.; Menchetti, L.; Bellucci, S.; Ranucci, D.; Marenzoni, M.L.; Franciosini, M.P. Antimicrobial susceptibility of Escherichia coli and ESBL-producing Escherichia coli diffusion in conventional, organic and antibiotic-free meat chickens at slaughter. Animals 2020, 10, 1215. [CrossRef]

33. Kizil, S.; Hasimi, N.; Tolan, V. Biological activities of Origanum, Satureja, Thymbra and Thymus species grown in Turkey. J. Essent. Oil Bear. Plants 2014, 17, 460-468. [CrossRef]

34. Altemimi, A.; Lakhssassi, N.; Baharlouei, A.; Watson, D.G.; Lightfoot, D.A. Phytochemicals: Extraction, isolation, and identification of bioactive compounds from plant extracts. Plants 2017, 6, 42. [CrossRef] [PubMed]

35. Ceruso, M.; Clement, J.A.; Todd, M.J.; Zhang, F.; Huang, Z.; Anastasio, A.; Pepe, T.; Liu, Y. The inhibitory effect of plant extracts on growth of the foodborne pathogen, Listeria monocytogenes. Antibiotics 2020, 9, 319. [CrossRef] [PubMed]

36. Chalchat, J.C.; Gorunović, M.S.; Maksimović, Z.A. Essential oil of Satureja kitaibelii Wierzb. f. aristata (Vand.) Hayek, Lamiaceae from Eastern Serbia. J. Essent. Oil Res. 1999, 11, 691-692. [CrossRef]

37. Kundaković, T.; Milenković, M.; Zlatković, S.; Kovačević, N.; Nikolić, G. Composition of Satureja kitaibelii essential oil and its antimicrobial activity. Nat. Prod. Commun. 2011, 6, 1353-1356. [CrossRef] [PubMed]

38. Slavkovska, V.; Jančić, R.; Bojović, S.; Milosavljević, S.; Đoković, D. Variability of essential oils of Satureja montana L., and Satureja kitaibelii Wierzb. ex Heuff. from the central part of the Balkan peninsula. Phytochemistry 2000, 57, 71-76. [CrossRef]

39. Milosavljević, P.N.; Veličković, T.D.; Ranđelović, V.N. Antibacterial activity and chemical composition of essential oil of savory (Satureja kitaibelii Wierzb. ex Heuff.). In Proceedings of the 6th Symposium on flora Southeastern Serbia, Sokobanja, Serbia, 4-7 July 2000; pp. 181-185.

40. Đorđević, A.; Palić, I.; Stojanović, G.; Ristić, N.; Palić, R. Chemical profile of Satureja kitaibelii Wierzb. ex Heuff. essential oils: Composition of Satureja kitaibelii essential oils. Int. J. Food Prop. 2014, 17, 2157-2165. [CrossRef]

41. Marchese, A.; Arciola, C.R.; Barbieri, R.; Silva, A.S.; Nabavi, S.F.; Sokeng, A.J.T.; Izadi, M.; Jafari, N.J.; Suntar, I.; Daglia, M.; et al. Update on monoterpenes as antimicrobial agents: A particular focus on $p$-cymene. Materials 2017, 10, 947. [CrossRef]

42. Reichling, J.; Suschke, U.; Schneele, J.; Geiss, H.K. Antibacterial activity and irritation potential of selected essential oil components-Structure-activity relationship. Nat. Prod. Commun. 2006, 1, 1003-1012. [CrossRef]

43. Li, Z.H.; Cai, M.; Liu, Y.S.; Sun, P.L.; Luo, S.L. Antibacterial activity and mechanisms of essential oil from Citrus medica L. var. sarcodactylis. Molecules 2019, 24, 1577. [CrossRef] 
44. Duman, A.D.; Telci, I.; Dayisoylu, K.S.; Digrak, M.; Demirtas, İ.; Almae, M.H. Evaluation of bioactivity of linalool-rich essential oils from Ocimum basilucum and Coriandrum sativum varieties. Nat. Prod. Commun. 2010, 5, 969-974. [CrossRef] [PubMed]

45. Al-Bayati, F.A. Synergistic antibacterial activity between Thymus vulgaris and Pimpinella anisum essential oils and methanol extracts. J. Ethnopharmacol. 2008, 116, 403-406. [CrossRef]

46. Kirillov, V.; Stikhareva, T.; Mukanov, B.; Chebotko, N.; Ryazantsev, O.; Atazhanova, G.; Adekenov, S. Composition of the essential oil of Thymus serpyllum L. from Northern Kazakhstan. J. Essent. Oil Bear. Plants 2016, 19, 212-222. [CrossRef]

47. Baczek, K.; Pioro-Jabrucka, E.; Kosakowska, O.; Weglarz, Z. Intraspecific variability of wild thyme (Thymus serpyllum L.) occurring in Poland. J. Appl. Res. Med. Aromat. Plants 2019, 12, 30-35. [CrossRef]

48. Nikolić, B.; Matović, M.; Mladenović, K.; Todosijević, M.; Stanković, J.; Đorđević, I.; Marin, P.; Tešević, V. Volatiles of Thymus serpyllum obtained by three different methods. Nat. Prod. Commun. 2019, 14, 1-3. [CrossRef]

49. Aćimović, M.; Cvetković, M.; Stanković, J.; Igić, R.; Todosijević, M.; Vuković, D.; Brašanac, D. Essential oil composition of the Thymus serpyllum L. from Kopaonik Mountain. J. Agron. Technol. Eng. Manag. 2019, 2, 241-247.

50. Stanisavljević, D.; Zlatković, B.; Ristić, M.; Veličković, D.; Đorđević, S.; Lazić, M. The chemical composition of the essential oil of (Thymus serpyllum) from Kopaonik Mountain. Adv. Technol. 2012, 1, 25-29.

51. Petrović, S.S.; Ristić, M.S.; Babović, N.V.; Lazić, M.L.; Francišković, M.; Petrović, S.D. Chemical composition and antioxidative activity of essential oil of Thymus serpyllum L. Hem. Ind. 2014, 68, 389-397. [CrossRef]

52. Raal, A.; Paaver, U.; Arak, E.; Orav, A. Content and composition of the essential oil of Thymus serpyllum L. growing wild in Estonia. Medicina (Kaunas) 2004, 40, 795-800.

53. Wesolowska, A.; Grzeszczuk, M.; Jadczak, D. Comparison of chemical compositions of essential oils isolated by hydrodistillation from wild thyme (Thymus serpyllum L.) with use of Deryng and Clevenger apparatus. Herba Pol. 2014, 60, 6. [CrossRef]

54. Wesolowska, A.; Grzeszczuk, M.D.; Jadczak, D.; Nawrotek, P.; Struk, M. Comparison of the chemical composition and antimicrobial activity of Thymus serpyllum essential oils. Not. Bot. Horti Agrobot. Cluj Napoca 2015, 43, 432-438. [CrossRef]

55. Verma, R.S.; ur Rahman, L.; Chanotiya, C.S.; Verma, R.K.; Singh, A.; Yadav, A.; Chauhan, A.; Yadav, A.K.; Singh, A.K. Essential oil composition of Thymus serpyllum cultivated in the Kumaon Region of Western Hymalaya, India. Nat. Prod. Commun. 2009, 4, 987-988. [PubMed]

56. Mašković, P.; Đurović, S.; Radojković, M.; Cvetanović, A.; Veličković, V.; Zeković, Z.; Miletić, N. Isolation and chemical profile of Thymus serpyllum L. and Lavandula angustifolia Mill. essential oil. In Proceedings of the 22th International Symposium on Biotechnology, Čačak, Serbia, 2017; pp. 541-548.

57. Aziz, S.; ur-Rehman, H.; Irshad, M.; Asghar, S.F.; Hussain, H.; Ahmed, I. Phytotoxic and antifungal activities of essential oils of Thymus serpyllum grown in the State of Jammu and Kashmir. J. Essent. Oil Bear. Plants 2010, 13, 224-229. [CrossRef]

58. Satyal, P.; Murray, B.L.; Mc Feeters, R.L.; Setzer, W.N. Essential oil characterization of Thymus vulgaris from various geographical locations. Foods 2016, 5, 70. [CrossRef]

59. Thompson, J.D.; Chalchat, J.C.; Michet, A.; Linhart, Y.B.; Ehlers, B. Qualitative and quantitative variation in monoterpene co-occurrence and composition in the essential oil of Thymus vulgaris chemotypes. J. Chem. Ecol. 2003, 29, 859-880. [CrossRef]

60. Bhattamisra, S.K.; Kuean, C.H.; Chieh, L.B.; Yan, V.L.Y.; Lee, C.K.; Hooi, L.P.; Shyan, L.P.; Liew, Y.K.; Candasamy, M.; Sahu, P.S. Antibacterial activity of geraniol in combination with standard antibiotics against Staphylococcus aureus, Escherichia coli and Helicobacter pylori. Nat. Prod. Commun. 2018, 13, 791-793. [CrossRef]

61. Gochev, V.; Wlcek, K.; Buchbauer, G.; Stoyanova, A.; Dobreva, A.; Schmidt, E.; Jirovetz, L. Comparative evaluation of antimicrobial activity and composition of rose oils from various geographic origins, in particular Bulgarian rose oil. Nat. Prod. Commun. 2008, 3, 1063-1068. [CrossRef]

62. Stešević, D.; Jaćimović, Ž.; Šatović, Z.; Šapčanin, A.; Jančan, G.; Kosović, M.; Damjanović-Vratnica, B. Chemical characterization of wild growing Origanum vulgare populations in Montenegro. Nat. Prod. Commun. 2018, 13, 1357-1362. [CrossRef]

63. Gonceariuc, M.; Balamus, Z.; Sandu, T.; Romanciuc, G.; Gonceariuc, N. Essential oil of Origanum vulgare ssp. vulgare L. and Origanum vulgare ssp. hirtum (Link) Ietswaart from Moldova: Content and chemical composition. Int. J. Agric. Innov. Res. 2014, 3, 659-663. 
64. Lukas, B.; Schmiderer, C.; Novak, J. Essential oil diversity of European Origanum vulgare L. (Lamiaceae). Phytochemistry 2015, 119, 32-40. [CrossRef]

65. Mockute, D.; Bernotiene, G.; Judzentiene, A. The essential oil of Origanum vulgare L. ssp. vulgare growing wild in Vilnius district (Lithuania). Phytochemistry 2001, 57, 65-69. [CrossRef]

66. Gong, H.Y.; Liu, W.H.; LV, G.Y.; Zhou, X. Analysis of essential oils of Origanum vulgare from six production areas of China and Pakistan. Rev. Bras. Farmacogn. 2014, 24, 25-32. [CrossRef]

67. Vazirian, M.; Mohammadi, M.; Faraei, M.H.; Amin, G.; Amanzadeh, Y. Chemical composition and antioxidant activity of Origanum vulgare subsp. vulgare essential oil from Iran. Res. J. Pharmacogn. 2015, 2, 41-46.

68. Andi, S.; Nazderi, V.; Hadian, J.; Zamani, Z. Variability of essential oil composition of Origanum vulgare L. ssp. vulgare populations from Iran. Med. Aromat. Plant Sci. Biotechnol. 2011, 5, 152-155.

69. Pirigharnaei, M.; Zare, S.; Heidary, R.; Khara, J.; Sabzi, R.E.; Kheiry, F. The essential oil compositions of Iranian oregano (Origanum vulgare L.) populations in field and provenance from Piranshahr district, West Azerbaijan province, Iran. Avicenna J. Phytomed. 2011, 1, 106-114.

70. Kosakowska, O.; Czupa, W. Morphological and Chemical variability of common oregano (Origanum vulgare L. subsp. vulgare) occurring in eastern Poland. Herba Pol. 2018, 64, 11-21. [CrossRef]

71. Sezik, E.; Tumen, G.; Kirimer, N.; Ozek, T.; Baser, K.H.C. Essential oil composition of four Origanum vulgare subspecies of Anatolian origin. J. Essent. Oil Res. 1993, 5, 425-431. [CrossRef]

72. Moghadam, A.R.L. Chemical composition of the essential oils of Origanum vulgare. Bull. Environ. Pharmacol. Life Sci. 2015, 4, 150-152.

73. Chrishti, S.; Kaloo, Z.; Sultan, P.; Nisa, H.T.; Nisa, N.T. Quantitative analysis of the essential oil of Origanum vulgare (L) growing in the Kashmir valley. Int. J. Appl. Res. 2016, 2, 552-557.

74. Radusiene, J.; Stankevičiene, D.; Venskutonis, R. Morphological and chemical variation of Origanum vulgare L. from Lithuania. Acta Hortic. 2005, 675, 197-203.

75. Ličina, B.Z.; Stefanović, O.D.; Vasić, S.M.; Radojević, I.D.; Dekić, M.S.; Čomić, L.R. Biological activities of the extracts from wild growing Origanum vulgare L. Food Control 2013, 33, 498-504. [CrossRef]

76. Stanojević, L.P.; Stanojević, J.S.; Cvetković, D.J.; Ilić, D.P. Antioxidant activity of oregano essential oil (Origanum vulgare L.). Biol. Nyssana 2016, 7, 131-139.

77. Simões, M.; Rocha, S.; Coimbra, M.A.; Vieira, M.J. Enhancement of Escherichia coli and Staphylococcus aureus antibiotic susceptibility using sesquiterpenoids. Med. Chem. 2008, 4, 616-623. [CrossRef] [PubMed]

78. Villecco, M.B.; Catalán, J.V.; Vega, M.I.; Garibotto, F.M.; Enriz, R.D.; Catalán, C.A.N. Synthesis and antibacterial activity of highly oxygenated 1,8-Cineole derivatives. Nat. Prod. Commun. 2008, 3, 303-312. [CrossRef]

79. Stević, T.; Šavikin, K.; Ristić, M.; Zdunić, G.; Janković, T.; Krivokuća-Đokić, D.; Vulić, T. Composition and antimicrobial activity of the essential oil of the leaves of black currant (Ribes nigrum L.) cultivar Čačanska crna. J. Serb. Chem. Soc. 2010, 75, 35-43. [CrossRef]

80. Borges, A.; Lopez-Romero, J.C.; Oliveira, D.; Giaouris, E.; Simoes, M. Prevention, removal and inactivation of Escherichia coli and Staphylococcus aureus biofilms using selected monoterpenes of essential oils. J. Appl. Microbiol. 2017, 123, 104-115. [CrossRef]

81. Judzentiene, A. Atypical chemical profiles of wild yarrow (Achillea millefolium L.) essential oils. Rec. Nat. Prod. 2016, 10, 262-268.

82. Radulović, N.; Zlatković, B.; Palić, R.; Stojanović, G. Chemotaxonomic significance of the Balkan Achillea volatiles. Nat. Prod. Commun. 2007, 2, 453-474. [CrossRef]

83. Nadim, M.M.; Malik, A.A.; Ahmad, J.; Bakshi, S.K. The essential oil composition of Achillea millefolium L. cultivated under tropical condition in India. World J. Agric. Sci. 2011, 7, 561-565.

84. Pljevljakušić, D.; Ristić, M.; Šavikin, K. Screening of yarrow (Achillea millefolium Agg.) populations in Serbia for yield components and essential oil composition. Lek. Sirovine 2017, 37, 25-32. [CrossRef]

85. Mockute, D.; Judzentiene, A. Chemotypes of the essential oils of Achillea millefolium L. ssp. millefolium growing wild in Eastern Lithuania. Chemija 2002, 13, 168-173.

86. Tuberoso, C.; Kowalczyk, A. Chemical composition of the essential oils of Achillea millefolium L. isolated by different distillation methods. J. Essent. Oil Res. 2009, 21, 108-111. [CrossRef]

87. Mazandarani, M.; Mirdeilami, S.Z.; Pessarakli, M. Essential oil composition and antibacterial activity of Achillea millefolium L. from different regions in North east of Iran. J. Med. Plant Res. 2013, 7, 1063-1069. 
88. Candan, F.; Unlu, M.; Tepe, B.; Daferera, D.; Polissiou, M.; Sökmen, A.; Akpulat, H.A. Antioxidant and antimicrobial activity of the essential oil and methanol extracts of Achillea millefolium subsp. millefolium Afan. (Asteraceae). J. Ethnopharmacol. 2003, 87, 215-220. [CrossRef]

89. El-Kalamouni, C.; Venskutonis, P.R.; Zebib, B.; Merah, O.; Raynaud, C.; Talou, T. Antioxidant and antimicrobial activities of the essential oil of Achillea millefolium L. grown in France. Medicines 2017, 4, 30. [CrossRef]

90. Dajić Stevanović, Z.; Pljevljakušić, D.; Ristić, M.; Šoštarić, I.; Kresovic, M.; Simić, I.; Vrbničanin, S. Essential oil composition of Achillea millefolium agg. populations collected from saline habitats in Serbia. Essent. Oil Bear. Plants 2015, 18, 1343-1352. [CrossRef]

91. Kocak, A.; Bagci, E.; Bakoglu, A. Chemical composition of essential oils of Achillea teterifolia Willd. and A. millefolium L. subsp. millefolium growing in Turkey. Asian J. Chem. 2010, 22, 3653-3658.

92. Raal, A.; Orav, A.; Arak, E. Essential oil content and composition in commercial Achillea millefolium L. herbs from different countries. J. Essent. Oil Bear. Plants 2012, 15, 22-31. [CrossRef]

93. Bączek, K.; Kosakowska, O.; Przybył, J.L.; Kuźma, P.; Ejdys, M.; Obiedziński, M.; Węglarz, Z. Intraspecific variability of yarrow (Achillea millefolium L. s.l.) in respect of developmental and chemical traits. Herba Pol. 2015, 61, 37-52. [CrossRef]

94. Bozin, B.; Mimica-Dukic, N.; Bogavac, M.; Suvajdzic, L.; Simin, N.; Samojlik, I.; Couladis, M. Chemical composition, antioxidant and antibacterial properties of Achillea collina Becker ex Heimerl s.l. and A. pannonica Scheele essential oils. Molecules 2008, 13, 2058-2068. [CrossRef]

95. Chalchat, J.C.; Petrovic, S.D.; Maksimovic, Z.A.; Gorunovic, M.S. Aromatic plants of Yugoslavia. III. Chemical composition of essential oils of Achillea abrotanoides (Vis.) Vis., A. clypeolata Sibth. \& Sm., A. depressa Janka and A. stricta Schleicher et Koch. J. Essent. Oil Res. 2005, 17, 549-552.

96. Simic, N.; Palić, R.; Randjelovic, V. Composition and antibacterial activity of Achillea clypeolata essential oil. Flavour Frag. J. 2004, 20, 127-130. [CrossRef]

97. Maffei, M.; Mucciarelli, M.; Scannerini, S. Essential oils from Achillea species of different geographic origin Biochem. System. Ecol. 1994, 22, 679-687. [CrossRef]

98. Cvetković, S.J.; Dimitrijević, V.M.; Ilić, D.M.; Simonović, R.S.; Stankov-Jovanović, P.V.; Mitić, D.V.; Stojanović, S.G. Antioxidant activity of Achillea clypeolata Sm. In Proceedings of the International Congress of Chemists and Chemical Engineers of Bosnia and Herzegovina, Sarajevo, Bosnia and Herzegovina, 10-12 October 2014.

99. Li, L.; Li, Z.W.; Yin, Z.Q.; Wei, Q.; Jia, R.Y.; Zhou, L.J.; Xu, J.; Song, X.; Zhou, Y.; Du, Y.H.; et al. Antibacterial activity of leaf essential oil and its constituents from Cinnamomum longepaniculatum. Int. J. Clin. Exp. Med. 2014, 7, 1721-1727. [PubMed]

100. European Pharmacopeia 9.0, 9th ed.; Council of Europe: Strasbourg, France, 2017; ISBN 978-9-2871-8133-6.

101. Aćimović, M.; Pezo, L.; Tešević, V.; Čabarkapa, I.; Todosijević, M. QSRR Model for predicting retention indices of Satureja kitaibelii Wierzb. ex Heuff. essential oil composition. Ind. Crop. Prod. 2020, 154, 112752. [CrossRef]

102. Harary, F. Graph Theory; Addison-Wesley: Reading, PA, USA, 1969; ISBN 9780201410334.

103. Ursem, R.; Tikunov, Y.; Bovy, A.; Berloo, R.; van Eeuwijk, F. A correlation network approach to metabolic data analysis for tomato fruits. Euphytica 2008, 161, 181-193. [CrossRef]

104. CLSI. Methods for Dilution Antimicrobial Susceptibility Tests for Bacteria that Grow Aerobically, 11th ed.; CLSI: Wayne, PA, USA, 2018.

105. Čabarkapa, I.; Čolović, R.; Đuragić, O.; Popović, S.; Kokić, B.; Milanov, D.; Pezo, L. Anti-biofilm activities of essential oils rich in carvacrol and thymol against Salmonella enteritidis. Biofouling 2019, 35, 361-375. [CrossRef]

Sample Availability: Samples of the compounds are not available from the authors.

Publisher's Note: MDPI stays neutral with regard to jurisdictional claims in published maps and institutional affiliations.

(C) 2020 by the authors. Licensee MDPI, Basel, Switzerland. This article is an open access article distributed under the terms and conditions of the Creative Commons Attribution (CC BY) license (http://creativecommons.org/licenses/by/4.0/). 